\title{
REWARD DISTRIBUTIONS ASSOCIATED WITH SOME BLOCK TRIDIAGONAL TRANSITION MATRICES WITH APPLICATIONS TO IDENTITY BY DESCENT
}

\author{
VALERI T. STEFANOV, ${ }^{*}$ The University of Western Australia \\ FRANK BALL, ${ }^{* *}$ University of Nottingham
}

\begin{abstract}
Markov and semi-Markov processes with block tridiagonal transition matrices for their embedded discrete-time Markov chains are underlying stochastic models in many applied probability problems. In particular, identity-by-descent (IBD) problems for uncle-type and cousin-type relationships fall into this class. More specifically, the exact distributions of relevant IBD statistics for two individuals in either an uncle-type or cousin-type relationship are of interest. Such statistics are the amount of genome shared IBD by the two related individuals on a chromosomal segment and the number of IBD pieces on such a segment. These lead to special reward distributions associated with block tridiagonal transition matrices for continuous-time Markov chains. A method is provided for calculating explicit, closed-form expressions for Laplace transforms of general reward functions for such Markov chains. Some calculation results on the cumulative probabilities of relevant IBD statistics via a numerical inversion of the Laplace transforms are also provided for uncle/nephew and first-cousin relationships.
\end{abstract}

Keywords: Semi-Markov process; stopping time; reward function; Laplace transform; genomic continuum model

2000 Mathematics Subject Classification: Primary 60E10

Secondary 92D10

\section{Introduction}

Markov and semi-Markov processes with block tridiagonal transition matrices for their embedded discrete-time Markov chains are underlying stochastic models in many applied probability problems. In particular, identity-by-descent (IBD) problems for uncle-type and cousin-type relationships fall into that class of problem. Although the motivation for this paper stems from such IBD problems, our results on block tridiagonal matrices might also be useful in other applied areas. The IBD of genetic material amongst individuals in a pedigree is a fundamental concept in genetics. Evaluations of the distributions of relevant IBD statistics are of interest in the framework of the genomic continuum model. Such statistics are the amount of genome shared IBD by two related individuals in a pedigree on a chromosomal segment and the number of corresponding IBD pieces on such a segment. There are few exact results in this area and they concern only grandparent-type, sib, and half-sib relationships; see Stefanov (2000a),

Received 7 February 2008; revision received 20 March 2009.

* Postal address: School of Mathematics and Statistics, The University of Western Australia, Crawley, WA 6009, Australia. Email address: stefanov@maths.uwa.edu.au

** Postal address: School of Mathematical Sciences, University of Nottingham, Nottingham, NG7 2RD, UK.

Email address: fgb@maths.nott.ac.uk 
(2002), (2004), Ball and Stefanov (2005), and Walters and Cannings (2005). Previously, only approximations to such distributions have been investigated, such as Poisson approximations (cf. Bickeboller and Thompson (1996a), (1996b)). Some simulation methods have also been suggested for such evaluations by Browning (2000), and a combination of analytical results with relevant simulations have been introduced in Cannings (2003). The uncle-type and cousin-type relationships are basic and important nonunilineal relationships. Note that two individuals are said to be in a unilineal relationship if one is a descendant of the other.

In this paper a method is provided for calculating explicit, closed-form expressions for Laplace transforms of general reward functions associated with some block tridiagonal transition matrices for continuous-time Markov chains. In particular, these cover relevant IBD distributions for uncle-type and cousin-type relationships. Successful numerical inversion of these transforms yields the corresponding cumulative probabilities. Some calculation results of cumulative probabilities are also provided for uncle/nephew and first-cousin relationships. More powerful PCs than those currently available should be able to handle the computational problems arising with numerical inversion of the Laplace transforms for high-order uncle-type and cousin-type relationships.

Our models are special cases of inhomogeneous quasi-birth-and-death (QBD) processes and there is a huge literature on these. There are many results on QBD processes that are somewhat related to ours (see Gaver et al. (1984), Lucantoni et al. (1994), Latouche and Ramaswami (1999), and Asmussen and Pihlsgård (2004)), including distributions for special rewards within first passage times (cf. Li and Cao (2004)). On the other hand, our explicit expressions for the Laplace transforms (Theorem 3 in Section 4) do not involve infinite sums or inverse matrices and, therefore, their evaluation does not create any computational problems.

Our methodology is different from that used in the literature on QBD processes and it treats them with semi-Markov tools. More details on the methodology follow. The mathematical model of the problem leads to evaluation of distributions of special reward functions associated with continuous-time Markov chains observed on fixed time intervals. Recent results of Stefanov (2006) provide closed-form solutions for two-dimensional Laplace transforms of the cumulative distribution functions of reward functions, on finite-time horizons, for Markov chains and semi-Markov processes. These solutions are practicable if the number of states of the embedded discrete-time Markov chain is small. More specifically, these solutions are expressed in terms of two-dimensional Laplace transforms for joint distributions associated with the first passage times between states, where the latter Laplace transforms are derivable explicitly as explained in Stefanov (2006). For the simple uncle/nephew and first-cousin relationships, the number of states is 4 and 7, respectively, and for great-uncle/great-nephew and second-cousin relationships these are 8 and 21 , respectively. The evaluations of the Laplace transforms of relevant joint distributions associated with the first passage times for such low-order relationships are implementable. For higher-order uncle-type and cousin-type relationships, we need to find a more practicable formula. We provide a method that leads to such a formula, which allows calculation of explicit, closed-form expressions for the Laplace transforms of the aforementioned IBD statistics. In particular, for any uncle-type relationship, the computations are associated with only 8- and 9-state embedded Markov chains and, for any cousin-type relationship, with 14- and 15-state Markov chains.

The paper is organized as follows. The motivation, as well as the underlying mathematical models for the uncle-type and cousin-type relationships, are discussed in the next section. Some preliminary results required in the sequel are found in Section 3. The main results are presented in Section 4. Applications to IBD distributions for uncle-type and cousin-type relationships 
are described in Section 5. A relevant extension of the result in Stefanov (2006) for rewards on semi-Markov processes, required in Section 4, is provided in Appendix A.

Throughout the paper, $\boldsymbol{I}$ is the identity matrix and $\mathbf{1}$ is a column vector of $1 \mathrm{~s}$, the dimensions of these being apparent from the context. Any sum is 0 if vacuous.

\section{Motivation}

\subsection{Identity by descent}

The genome of diploid organisms (such as humans) resides on a set of pairs of chromosomes. For example, humans have 23 pairs of chromosomes, 22 autosomal pairs and one pair of sex chromosomes (XX for females and XY for males). Any small segment of the DNA sequence on a chromosome is called a locus. In particular, genes are also called loci. A locus that exhibits observable variation in the DNA sequence is called a marker locus. The number of marker loci on each chromosome is huge and, therefore, the genome can be viewed as a continuum. That is, a chromosome is viewed as a finite interval and a locus simply indicates a particular position on that interval. For each pair of chromosomes of an individual, one chromosome is inherited from his father and the other from his mother. The chromosome inherited from a parent is either (i) the entire one from the grandfather (that is, the one the parent derived from his father), (ii) the entire one from the grandmother, or (iii) a combination of the two. In case (iii), the inherited chromosome consists of alternating pieces of the two grandparental chromosomes. The occurrences of switches (from a grandpaternal to a grandmaternal and vice versa) along a chromosome are usually modelled by a homogeneous Poisson process. The distances between loci are measured by the expected number of switches between them. The units of that distance are called morgans. Typical lengths of the human chromosome are between 0.5 and 3 morgans.

In the context of a grandparent/grandchild relationship, the DNA sequence at a locus from a chromosome of the grandchild is called identical by descent (IBD) with the DNA sequence at the same locus of the corresponding chromosome of the grandparent if the former DNA is inherited by the grandchild from the latter of the grandparent. Assume now that two individuals are in a half-sib relationship. Then the DNA sequence at a particular locus from the corresponding chromosomes of the two half-sibs is called IBD if that DNA sequence is inherited from the same grandparent. More generally, the DNA sequence at a locus for two related individuals is called IBD if the DNA sequence at that locus of one of these individuals is a physical copy of the DNA sequence at the same locus of the other individual (as in the grandparent/grandchild relationship above) or both are physical copies of the DNA sequence at the same locus of the corresponding chromosome in a common ancestor of the two individuals (as in the half-sib relationship above). For a given relationship, for example uncle/nephew, there are a finite number of parent/child relationships that affect IBD. The occurrences of switches along the inherited chromosomes for each such parent/child relationship are assumed to follow independent unit-rate Poisson processes. Thus, for each such parent/child relationship, we can derive a two-state continuoustime Markov chain, with states 0 and 1, corresponding to whether the DNA sequence at a particular location on the chromosome is inherited from the child's grandmother or grandfather, respectively. It follows that, if $m$ parent/child relationships impact on a given relationship then IBD for the given relationship is modelled via a continuous-time Markov chain, $X_{t}$ say, having state space $\{0,1\}^{m}$, given by the cartesian product of the $m$ independent parent/child Markov chains. However, there are symmetries present in $X_{t}$ which enable its state space to be substantially reduced. A detailed treatment of the concept of IBD and the relevant mathematical 
models for various relationships are discussed in Donnelly (1983). The relevant models for uncle-type and cousin-type relationships are introduced in the next subsection.

The motivation of this paper stems from the need to evaluate p-values in relevant tests associated with resemblance of individuals suspected to be in a particular relationship and p-values for tests associated with identifying chromosomal segments responsible for complex diseases. Stefanov (2000a), (2002) discussed such tests for the simple grandparent/grandchild and sib relationships based on continuous genome data. IBD distributional results are widely used on discrete genome data (few loci or, more recently, multiple loci) (see Thompson (2000) and Lange (2002)). However, owing to a lack of exact distributional results on the continuous genome beyond those for the simple grandparent/grandchild and sib relationships, such tests have not been devised. The aim of this paper is to provide such distributional results on the continuous genome for a large set of relationships (uncle-type and cousin-type). Then the p-values mentioned above are obtained from cumulative probabilities of the aforementioned IBD statistics, that is, the amount of genome shared IBD on chromosomal segments and the number of IBD pieces on such segments. In particular, our results yield p-values of relevant significance tests for resemblance of two individuals as being in a particular uncle-type (or cousin-type) relationship. Such tests are devised in the same way as is done in Stefanov (2000a), (2002) for the simple grandparent/grandchild and sib relationships.

\subsection{Uncle-type and cousin-type relationships}

First we describe the processes of interest in this paper. Let $\left(\hat{X}_{t}, \hat{Y}_{t}\right)$ be an inhomogeneous QBD chain with discrete-time parameter and state space $\{(i, j): i=0,1, \ldots, n-1, j=$ $1,2, \ldots, k\}$. Conventionally, $\hat{X}_{t}$ is called the level and $\hat{Y}_{t}$ the phase at time $t$. The number of phases $k$ is the same for all levels. The one-step transition probability matrices of such chains have a block tridiagonal structure and we consider the following special case of such matrices:

$$
\boldsymbol{B}_{n}=\left[\begin{array}{ccccc}
\boldsymbol{A} & (n-1) \operatorname{diag}[\boldsymbol{d}] & & & \\
\operatorname{diag}[\boldsymbol{d}] & \boldsymbol{A} & (n-2) \operatorname{diag}[\boldsymbol{d}] & & \\
& \ddots & \ddots & \ddots & \\
& & (n-2) \operatorname{diag}[\boldsymbol{d}] & \boldsymbol{A} & \operatorname{diag}[\boldsymbol{d}] \\
& & & (n-1) \operatorname{diag}[\boldsymbol{d}] & \boldsymbol{A}
\end{array}\right],
$$

where $\boldsymbol{A}$ is a matrix of order $k, \boldsymbol{d}=\left(d_{1}, d_{2}, \ldots, d_{k}\right)^{\top}$ is a $k$-dimensional vector, and $\operatorname{diag}[\boldsymbol{d}]$ is the diagonal $k \times k$ matrix with $d_{i}$ on the diagonal, that is,

$$
\operatorname{diag}[\boldsymbol{d}](i, j)= \begin{cases}d_{i} & \text { if } i=j, \\ 0 & \text { otherwise }\end{cases}
$$

The notation $\left(\hat{X}_{t}, \hat{Y}_{t}\right) \mid \boldsymbol{B}_{n}$ is also used for this QBD chain.

Throughout the paper, the models of interest, denoted by $\left(X_{t}, Y_{t}\right) \mid \boldsymbol{B}_{n}$, are semi-Markov processes whose embedded discrete-time Markov chains are the QBD chains $\left(\hat{X}_{t}, \hat{Y}_{t}\right) \mid \boldsymbol{B}_{n}$. Whenever such a semi-Markov process is considered, the relevant holding time distributions are also described. In particular, recall that continuous-time Markov chains are semi-Markov processes with exponentially distributed holding times.

The motivation for considering these models stems from genetics problems associated with evaluation of relevant distributions concerning the amount of genetic material shared in common by related individuals. More specifically, we are interested in uncle-type and cousin-type relationships. The underlying mathematical model (see Donnelly (1983)) for uncle-type and 
cousin-type relationships is $\left(X_{t}, Y_{t}\right) \mid \boldsymbol{B}_{n}$, with the following assumptions on the number of phases $k$, the matrix $\boldsymbol{B}_{n}$, and the holding time distributions.

Uncle-type relationships: $k=4 ; d_{1}=d_{3}=1 /(n+4), d_{2}=d_{4}=1 /(n+3)$; and

$$
\boldsymbol{A}=\left[\begin{array}{cccc}
0 & \frac{2}{n+3} & 0 & \frac{2}{n+3} \\
\frac{2}{n+4} & 0 & \frac{2}{n+4} & \frac{1}{n+4} \\
0 & \frac{2}{n+3} & 0 & \frac{2}{n+3} \\
\frac{2}{n+4} & \frac{1}{n+4} & \frac{2}{n+4} & 0
\end{array}\right] .
$$

The holding times are exponentially distributed with parameters $\left(\lambda_{1}, \lambda_{2}, \lambda_{3}, \lambda_{4}\right)$ for the four phases at each level, where $\lambda_{1}=\lambda_{3}=n+3$ and $\lambda_{2}=\lambda_{4}=n+4$. For example, $n=1$ corresponds to the uncle/nephew relationship, $n=2$ corresponds to the great-uncle/greatnephew relationship, etc. That is, the number of levels $n$ indicates the generation gap.

Cousin-type relationships: $k=7 ; d_{i}=1 /(n+5)$ for each $i=1,2, \ldots, 7$; and

$$
\boldsymbol{A}=\left[\begin{array}{ccccccc}
0 & \frac{2}{n+5} & \frac{2}{n+5} & 0 & \frac{2}{n+5} & 0 & 0 \\
\frac{2}{n+5} & 0 & 0 & \frac{2}{n+5} & 0 & \frac{2}{n+5} & 0 \\
\frac{2}{n+5} & 0 & 0 & \frac{2}{n+5} & 0 & \frac{2}{n+5} & 0 \\
0 & \frac{2}{n+5} & \frac{2}{n+5} & 0 & 0 & 0 & \frac{2}{n+5} \\
\frac{2}{n+5} & 0 & 0 & 0 & 0 & \frac{4}{n+5} & 0 \\
0 & \frac{1}{n+5} & \frac{1}{n+5} & 0 & \frac{2}{n+5} & 0 & \frac{2}{n+5} \\
0 & 0 & 0 & \frac{2}{n+5} & 0 & \frac{4}{n+5} & 0
\end{array}\right]
$$

The holding times are exponentially distributed with parameters $\lambda_{i}=n+5, i=1,2, \ldots, 7$, for the seven phases at each level. For example, $n=1$ corresponds to first cousins, $n=2$ corresponds to first cousins once removed, and $n=3$ corresponds to second cousins.

The initial probability vector for both types of relationship has the form

$$
\frac{1}{2^{n-1}}\left(\left(\begin{array}{c}
n-1 \\
0
\end{array}\right) \boldsymbol{u},\left(\begin{array}{c}
n-1 \\
1
\end{array}\right) \boldsymbol{u}, \ldots,\left(\begin{array}{c}
n-1 \\
n-1
\end{array}\right) \boldsymbol{u}\right)^{\top}
$$

where the vector $\boldsymbol{u}$ is equal to $\left(\frac{1}{4}, \frac{1}{4}, \frac{1}{4}, \frac{1}{4}\right)^{\top}$ and $\left(\frac{1}{8}, \frac{1}{8}, \frac{1}{8}, \frac{1}{8}, \frac{1}{8}, \frac{1}{4}, \frac{1}{8}\right)^{\top}$ for the uncle-type and cousin-type relationships, respectively.

The lengths of chromosomes can be viewed as time intervals, $[0, T]$ say for a chromosome of length $T$ morgans, where a locus on a chromosome corresponds to a time epoch. When a 
chromosome from one individual is aligned with the corresponding one of another individual, related to him, at each location (time epoch) we have two states: either the DNA sequence is IBD or it is not. Therefore, the IBD status along a chromosome can be viewed as a two-state process. Donnelly (1983) explained that for uncle-type and cousin-type relationships this twostate process can be embedded in the aforementioned $\left(X_{t}, Y_{t}\right) \mid \boldsymbol{B}_{n}$ processes as follows. The first two states of $\left(X_{t}, Y_{t}\right) \mid \boldsymbol{B}_{n}$ correspond to IBD status and the remaining states correspond to non-IBD status. Therefore, the following two reward functions for $\left(X_{t}, Y_{t}\right) \mid \boldsymbol{B}_{n}$ are of practical importance here: the time spent in the first two states within a time interval of length $T$ and the number of visits to the first two states within such a time interval. The genetic interpretation of these is as follows. The first reward function represents the amount of genome shared IBD by the two related individuals along a chromosomal segment of length $T$ morgans and the second reward function gives the number of IBD pieces within such a chromosomal segment.

\section{Preliminary results}

In this section we list a few relevant results on absorbing Markov chains and we derive a result (Lemma 1) on the state space restriction of semi-Markov processes. Lemma 1 is frequently used in the next section. Throughout the paper, the entries of a matrix $\boldsymbol{A}$ are written as $a_{i, j}$ or as $\boldsymbol{A}(i, j)$. The Laplace transform of a random variable $X$ is denoted by $\mathcal{L}[X](s)=\mathrm{E}\left(\mathrm{e}^{-s X}\right)$. Consider the following QBD transition probability matrix with $k$ phases and two levels:

$$
\boldsymbol{B}=\left[\begin{array}{cc}
\boldsymbol{Q} & \operatorname{diag}[\boldsymbol{x}] \\
\operatorname{diag}[\boldsymbol{y}] & \boldsymbol{R}
\end{array}\right]
$$

where $\boldsymbol{Q}$ and $\boldsymbol{R}$ are $k \times k$ matrices, and $\boldsymbol{x}=\left(x_{1}, \ldots, x_{k}\right)^{\top}$ and $\boldsymbol{y}=\left(y_{1}, \ldots, y_{k}\right)^{\top}$ are $k$-dimensional vectors. Some notation concerning relevant probability generating functions associated with absorbing finite-state Markov chains follows. Denote by $\boldsymbol{R} \mid \boldsymbol{y}$ the $2 k \times 2 k$ matrix

$$
\boldsymbol{R} \mid \boldsymbol{y}=\left[\begin{array}{cc}
\boldsymbol{R} & \operatorname{diag}[\boldsymbol{y}] \\
\mathbf{0} & \boldsymbol{I}
\end{array}\right],
$$

where $\mathbf{0}$ is a $k \times k$ matrix of 0 s. Assume that $\boldsymbol{R} \mid \boldsymbol{y}$ is the one-step transition probability matrix of a discrete-time Markov chain with $2 k$ states. Let $\tau_{r}$ be the time until absorption in the last $k$ states, given that the initial state is $r, 1 \leq r \leq k$, and let $\boldsymbol{T}_{r}(i, j)$ be the number of one-step transitions from state $i$ to state $j$, where $1 \leq i \leq k$ (the transient states) and $1 \leq j \leq 2 k$, by time $\tau_{r}$. Likewise, let $\boldsymbol{T}_{r}^{(n)}(i, j)$ be the count of $\boldsymbol{T}_{r}(i, j)$ given that absorption occurs in state $n+k$. Denote by $g_{\boldsymbol{R}} \mid \boldsymbol{y}, r(\boldsymbol{S}, \boldsymbol{s})$ the joint probability generating function of the $\boldsymbol{T}_{r}(i, j)$ until absorption, given that the initial state is $r, 1 \leq r \leq k$, that is,

$$
g_{\boldsymbol{R} \mid \boldsymbol{y}, r}(\boldsymbol{S}, \boldsymbol{s})=\mathrm{E}\left(\prod_{i, j=1}^{k} s_{i, j}^{\boldsymbol{T}_{r}(i, j)} \prod_{i=1}^{k} s_{i}^{\boldsymbol{T}_{r}(i, i+k)}\right),
$$

where the $s_{i, j}$ are the entries of the matrix $S$ and $s=\left(s_{1}, s_{2}, \ldots, s_{k}\right)^{\top}$. Of course,

$$
\sum_{i=1}^{k} \boldsymbol{T}_{r}(i, i+k)=1
$$

and if $\boldsymbol{T}_{r}(i, i+k)=1$ then absorption occurs through a one-step transition from state $i$ to state $i+k$. Denote by $g_{\boldsymbol{R}} \mid \boldsymbol{y}, r, j(\boldsymbol{S}, \boldsymbol{s})$ the conditional joint probability generating function of 
the $\boldsymbol{T}_{r}(i, j)$ until absorption at state $j+k$, that is,

$$
g_{\boldsymbol{R} \mid \boldsymbol{y}, r, j}\left(\boldsymbol{S}, s_{j}\right)=\mathrm{E}\left(s_{j} \prod_{n, m=1}^{k} s_{n, m}^{\boldsymbol{T}_{r}^{(n, m)}} \mid \boldsymbol{T}_{r}(j, j+k)=1\right)=\mathrm{E}\left(s_{j} \prod_{n, m=1}^{k} s_{n, m}^{\boldsymbol{T}_{r}^{(j)}(n, m)}\right) .
$$

Let $\beta_{i, j}(\boldsymbol{R} \mid \boldsymbol{y})$ be the probability that the Markov chain is absorbed at state $j+k, 1 \leq j \leq k$, given that the initial state is $i, 1 \leq i \leq k$. We denote by $\boldsymbol{B}(\boldsymbol{R} \mid \boldsymbol{y})$ the matrix whose entries are $\beta_{i, j}(\boldsymbol{R} \mid \boldsymbol{y})$. For the matrix of absorption probabilities, $\boldsymbol{B}(\boldsymbol{R} \mid \boldsymbol{y})$, and the probability generating functions, $g_{\boldsymbol{R} \mid \boldsymbol{y}, r}(\boldsymbol{S}, \boldsymbol{s})$ and $g_{\boldsymbol{R} \mid \boldsymbol{y}, r, j}\left(\boldsymbol{S}, s_{j}\right)$, we have (here $\boldsymbol{e}_{j}=(0, \ldots, 0,1,0, \ldots, 0)^{\top}$ is the $k \times 1$ unit vector with 1 in the $j$ th position and $\mathbf{1 1}^{\top}$ is a $k \times k$ matrix of $1 \mathrm{~s}$ )

$$
\begin{gathered}
\boldsymbol{B}(\boldsymbol{R} \mid \boldsymbol{y})=(\boldsymbol{I}-\boldsymbol{R})^{-1} \operatorname{diag}[\boldsymbol{y}], \\
\boldsymbol{g}_{\boldsymbol{R} \mid \boldsymbol{y}}(\boldsymbol{S}, \boldsymbol{s})=(\boldsymbol{I}-\boldsymbol{R}(\boldsymbol{S}))^{-1} \operatorname{diag}[\boldsymbol{y}] \boldsymbol{s}, \\
g_{\boldsymbol{R} \mid \boldsymbol{y}, r, j}\left(\boldsymbol{S}, s_{j}\right)=\frac{g_{\boldsymbol{R} \mid \boldsymbol{y}, r}\left(\boldsymbol{S}, s_{j} \boldsymbol{e}_{j}\right)}{g_{\boldsymbol{R} \mid \boldsymbol{y}, r}\left(\mathbf{1 1}^{\top}, \boldsymbol{e}_{j}\right)}, \quad r, j=1,2, \ldots, k,
\end{gathered}
$$

where $\boldsymbol{g}_{\boldsymbol{R} \mid \boldsymbol{y}}(\boldsymbol{S}, \boldsymbol{s})=\left(g_{\boldsymbol{R} \mid \boldsymbol{y}, 1}(\boldsymbol{S}, \boldsymbol{s}), g_{\boldsymbol{R} \mid \boldsymbol{y}, 2}(\boldsymbol{S}, \boldsymbol{s}), \ldots, g_{\boldsymbol{R} \mid \boldsymbol{y}, k}(\boldsymbol{S}, \boldsymbol{s})\right)^{\top}$ and the $(i, j)$ th entry of the $k \times k$ matrix $\boldsymbol{R}(\boldsymbol{S})$ is given by $\boldsymbol{R}(i, j) \boldsymbol{S}(i, j)\left(=\boldsymbol{R}(i, j) s_{i, j}\right)$. The first two identities in (3) follow from Iosifescu (1980, p. 103) (cf. also Karlin and Taylor (1981, p. 29)) and the third identity is an easy exercise. Note that the denominator in the third identity above is equal to the absorption probability, given that the initial state is $r$ and absorption occurs through a transition from state $j$ to state $j+k$, that is,

$$
\beta_{r, j}(\boldsymbol{R} \mid \boldsymbol{y})=g_{\boldsymbol{R} \mid \boldsymbol{y}, r}\left(\mathbf{1 1}^{\top}, \boldsymbol{e}_{j}\right) .
$$

Consider the semi-Markov process $\left(X_{t}, Y_{t}\right) \mid \boldsymbol{B}$, where $\boldsymbol{B}$ (see (2)) is the one-step transition probability matrix of the embedded discrete-time Markov chain and the Laplace transforms (LTs) of the holding (sojourn) times are described below. Also, recall that we denoted the embedded discrete-time Markov chain of $\left(X_{t}, Y_{t}\right) \mid \boldsymbol{B}$ by $\left(\hat{X}_{t}, \hat{Y}_{t}\right) \mid \boldsymbol{B}$. The LT of the holding time associated with a one-step transition from phase $i$ to phase $j$ within level $m(m=1,2)$ is denoted by $\rho_{i, j}^{(m)}(s)$, from phase $i$ in level 1 to phase $i$ in level 2 is denoted by $\rho_{i}^{(1,2)}(s)$, and likewise from phase $i$ in level 2 to phase $i$ in level 1 is denoted by $\rho_{i}^{(2,1)}(s)$. Recall from the matrix $\boldsymbol{B}$ that one-step transitions are not possible between states whose phases and levels are both different. Denote by $\left(\tilde{X}_{t}, \tilde{Y}_{t}\right) \mid \boldsymbol{B}$ the semi-Markov process derived from the semiMarkov process $\left(X_{t}, Y_{t}\right) \mid \boldsymbol{B}$ through restricting the state space to the states from level 1. More precisely, assuming that $X_{0}=1$, for $t>0$, if $X_{t}=1$ then $\left(\tilde{X}_{t}, \tilde{Y}_{t}\right)\left|\boldsymbol{B}=\left(X_{t}, Y_{t}\right)\right| \boldsymbol{B}$, whilst if $X_{t}=2$ then $\left(\tilde{X}_{t}, \tilde{Y}_{t}\right)\left|\boldsymbol{B}=\left(X_{u-}, Y_{u_{-}}\right)\right| \boldsymbol{B}$, where $u=\sup \left\{v<t: X_{v}=1\right\}$ and, for example, $X_{u-}=\lim _{v \uparrow u} X(v)$. Since $\tilde{X}_{t}$ is fixed (equal to 1 ), we may ignore it and say that $\tilde{Y}_{t} \mid \boldsymbol{B}$ is a semi-Markov process with $k$ states. Denote by $v_{i, j}$ the holding time in state $i$, given that the next state to be visited is state $j$, of the semi-Markov process $\tilde{Y}_{t} \mid \boldsymbol{B}$.

Lemma 1. The Laplace transform of $v_{i, j}, i, j=1, \ldots, k$, is given by

$$
\mathcal{L}\left[v_{i, j}\right](s)=\frac{q_{i, j} \rho_{i, j}^{(1)}(s)+x_{i} \beta_{i, j}(\boldsymbol{R} \mid \boldsymbol{y}) \rho_{i}^{(1,2)}(s) g_{\boldsymbol{R} \mid \boldsymbol{y}, i, j}\left(\mathcal{R}(s), \rho_{j}^{(2,1)}(s)\right)}{q_{i, j}+x_{i} \beta_{i, j}(\boldsymbol{R} \mid \boldsymbol{y})},
$$

where the entries of the $k \times k$ matrix $\mathcal{R}(s)$ are $\rho_{n, m}^{(2)}(s)$. The one-step transition probabilities of the embedded discrete-time Markov chain of the semi-Markov process $\tilde{Y}_{t} \mid \boldsymbol{B}$ are denoted 
by $\tilde{p}_{i, j}$ for the transition from state $i$ to state $j$ and given by

$$
\tilde{p}_{i, j}=q_{i, j}+x_{i} \beta_{i, j}(\boldsymbol{R} \mid \boldsymbol{y}), \quad i, j=1,2, \ldots, k,
$$

where recall that the $q_{i, j}$ are the entries of the matrix $\boldsymbol{Q}$ and $\beta_{i, j}(\boldsymbol{R} \mid \boldsymbol{y})$ is the absorption probability in state $j+k$ of a discrete-time Markov chain with transition probability matrix $\boldsymbol{R} \mid \boldsymbol{y}$ and initial state $i$.

Proof. A one-step transition from state $i$ to state $j$ in the embedded discrete-time Markov chain of the semi-Markov process $\tilde{Y}_{t} \mid \boldsymbol{B}$ may occur in two ways: either (i) through a direct transition from $(1, i)$ to $(1, j)$ in $\left(\hat{X}_{t}, \hat{Y}_{t}\right) \mid \boldsymbol{B}$ (with probability $q_{i, j} /\left(q_{i, j}+x_{i} \beta_{i, j}(\boldsymbol{R} \mid \boldsymbol{y})\right)$ ), or (ii) after a transition to level 2 and a subsequent excursion there before entering level 1 through state $(2, j)$ (with probability $\left.x_{i} \beta_{i, j}(\boldsymbol{R} \mid \boldsymbol{y}) /\left(q_{i, j}+x_{i} \beta_{i, j}(\boldsymbol{R} \mid \boldsymbol{y})\right)\right)$. Therefore, in view of the basic properties of semi-Markov processes, the distribution of the holding time $v_{i, j}$, denoted by $D_{v_{i, j}}$, is a mixture of two distributions, say $D_{1}$ and $D_{2}$, that is,

$$
D_{v_{i, j}}=\frac{q_{i, j}}{q_{i, j}+x_{i} \beta_{i, j}(\boldsymbol{R} \mid \boldsymbol{y})} D_{1}+\frac{x_{i} \beta_{i, j}(\boldsymbol{R} \mid \boldsymbol{y})}{q_{i, j}+x_{i} \beta_{i, j}(\boldsymbol{R} \mid \boldsymbol{y})} D_{2} .
$$

Here the LT of the distribution $D_{1}$ is $\rho_{i, j}^{(1)}(s)$. The distribution $D_{2}$ is equal to that of a sum of two independent random variables, of which the LT of the first one is $\rho_{i}^{(1,2)}(s)$ and the second one has the distribution of the excursion of the semi-Markov process $\left(X_{t}, Y_{t}\right) \mid \boldsymbol{B}$ in level 2 given that the initial phase is $i$, and given that the phase before leaving level 2 is $j$. This excursion equals the following sum of random sums: $\sum_{n, m=1}^{k} \sum_{r=1}^{\boldsymbol{T}_{i}^{(j)}(m, n)} Z_{n, m, r}$, where the $Z_{n, m, r}$ are independent and, for each pair $(n, m)$ and $r=1,2, \ldots$, the LT of $Z_{n, m, r}$ is $\rho_{n, m}^{(2)}(s)$; recall that, for $1 \leq i, j \leq k, \boldsymbol{T}_{i}^{(j)}(n, m)$ denotes the number of one-step transitions from state $n$ to state $m$ until absorption in a discrete-time Markov chain whose one-step transition matrix is given by $\boldsymbol{R} \mid \boldsymbol{y}$, given that the initial state is $i$ and absorption occurs in state $j+k$. Therefore, in view of Lemma 2, below, the LT of this excursion is given by

$$
g_{\boldsymbol{R} \mid \boldsymbol{y}, r, j}\left(\mathcal{R}(s), \rho_{j}^{(2,1)}(s)\right),
$$

where the conditional joint probability generating function $g_{\boldsymbol{R}} \mid \boldsymbol{y}, r, j\left(\boldsymbol{S}, s_{j}\right)$ has been introduced earlier. Thus, (4) follows from (6) and (7). It is easy to see that (5) holds. This completes the proof.

Lemma 2. Let $\boldsymbol{\Gamma}=\left(\gamma_{1}, \gamma_{2}, \ldots, \gamma_{r}\right)^{\top}$ be a vector of nonnegative integer-valued random variables with joint probability generating function $g_{\Gamma}\left(s_{1}, s_{2}, \ldots, s_{r}\right)$. Let $Z_{n}^{(i)}, i=1,2, \ldots, r$, be $r$ independent sequences of independent and identically distributed random variables that are independent of $\boldsymbol{\Gamma}$. For each $n$, it is assumed that the distribution of $Z_{n}^{(i)}$ follows the distribution of a random variable, $Z^{(i)}$ say, for $i=1,2, \ldots, r$. Let

$$
S_{i}=\sum_{j=1}^{\gamma_{i}} Z_{j}^{(i)}, \quad i=1,2, \ldots, r .
$$

Then the Laplace transform of the $r$-dimensional vector $\boldsymbol{S}=\left(S_{1}, \ldots, S_{r}\right)^{\top}$ is equal to

$$
\mathcal{L}[S]\left(t_{1}, t_{2}, \ldots, t_{r}\right)=g_{\Gamma}\left(\mathcal{L}\left[Z^{(1)}\right]\left(t_{1}\right), \mathcal{L}\left[Z^{(2)}\right]\left(t_{2}\right), \ldots, \mathcal{L}\left[Z^{(r)}\right]\left(t_{r}\right)\right),
$$


and, more generally, the Laplace transform of the $2 r$-dimensional vector $(\boldsymbol{S}, \boldsymbol{\Gamma})$ is equal to

$$
\begin{aligned}
\mathcal{L}[\boldsymbol{S}, \boldsymbol{\Gamma}]\left(t_{1}, t_{2}, \ldots, t_{2 r}\right) \\
\quad=g_{\boldsymbol{\Gamma}}\left(\exp \left\{-t_{r+1}\right\} \mathcal{L}\left[Z^{(1)}\right]\left(t_{1}\right), \exp \left\{-t_{r+2}\right\} \mathcal{L}\left[Z^{(2)}\right]\left(t_{2}\right) \ldots, \exp \left\{-t_{2 r}\right\} \mathcal{L}\left[Z^{(r)}\right]\left(t_{r}\right)\right) .
\end{aligned}
$$

Proof. The proof follows from Lemma 2.1 of Stefanov (2000b).

\section{Main results}

\subsection{State space restriction}

In this subsection we provide an effective route, via relevant recurrence relations, for the evaluation of explicit, closed-form expressions of the holding time distributions and transition probabilities of a state space restricted semi-Markov process. The latter is the semi-Markov process derived from $\left(X_{t}, Y_{t}\right) \mid \boldsymbol{B}_{n}$ (introduced in Section 2) via a state space restriction to the states in level 0 . To understand how the recurrence relations are derived, we consider first the special cases $n=2$ and $n=3$. The general case is stated in Theorem 1, below.

The $n=2$ case. Consider the process $\left(X_{t}, Y_{t}\right) \mid \boldsymbol{B}_{2}$. The holding times are exponentially distributed (that is, the process is a continuous-time Markov chain) and their parameters are given by $\lambda_{1}, \lambda_{2}, \ldots, \lambda_{k}$ for the $k$ phases at each of the two levels (recall that the levels are 0 and 1). Denote by $\left(\tilde{X}_{t}, \tilde{Y}_{t}\right) \mid \boldsymbol{B}_{2}$ the semi-Markov process derived from the continuous-time Markov chain $\left(X_{t}, Y_{t}\right) \mid \boldsymbol{B}_{2}$ through state space restriction of the latter to the states in level 0 . Since $\tilde{X}_{t} \mid \boldsymbol{B}_{2}$ is fixed (equal to 0), we may ignore it and say that $\tilde{Y}_{t} \mid \boldsymbol{B}_{2}$ is a semi-Markov process with $k$ states. Denote by $v_{i, j}^{(1)}$ the holding time in state $i$, given that the next state to be visited is $j$, of the semi-Markov process $\tilde{Y}_{t} \mid \boldsymbol{B}_{2}$.

Lemma 3. The Laplace transform of $v_{i, j}^{(1)}, i, j=1,2, \ldots, k$, is given by

$$
\mathcal{L}\left[v_{i, j}^{(1)}\right](s)=\frac{\lambda_{i}}{\lambda_{i}+s}\left(\frac{a_{i, j}+d_{i} \beta_{i, j}(\boldsymbol{A} \mid \boldsymbol{d}) g_{\boldsymbol{A} \mid \boldsymbol{d}, i, j}\left(\boldsymbol{\Lambda}(s), \lambda_{j} /\left(\lambda_{j}+s\right)\right)}{a_{i, j}+d_{i} \beta_{i, j}(\boldsymbol{A} \mid \boldsymbol{d})}\right),
$$

where the $(n, m)$ th entry of the $k \times k$ matrix $\boldsymbol{\Lambda}(s)$ is given by $\lambda_{n} /\left(\lambda_{n}+s\right)$. The one-step transition probabilities of the embedded discrete-time Markov chain of $\tilde{Y}_{t} \mid \boldsymbol{B}_{2}$ are denoted by $\tilde{p}_{i, j}^{(1)}$ and given by

$$
\tilde{p}_{i, j}^{(1)}=a_{i, j}+d_{i} \beta_{i, j}(\boldsymbol{A} \mid \boldsymbol{d}), \quad i, j=1,2, \ldots, k ;
$$

recall that $\beta_{i, j}(\boldsymbol{A} \mid \boldsymbol{d})$ is the absorption probability in state $j+k$ of a discrete-time Markov chain with transition probability matrix $\boldsymbol{A} \mid \boldsymbol{d}$ and initial state $i$.

Proof. The result follows from Lemma 1, replacing $\boldsymbol{B}$ by $\boldsymbol{B}_{2}$ and, for $i, j=1,2, \ldots, k$, replacing $\rho_{i, j}^{(1)}(s), \rho_{i, j}^{(2)}(s), \rho_{i}^{(1,2)}(s)$, and $\rho_{i}^{(2,1)}(s)$ by the LT of an exponentially distributed random variable whose parameter is $\lambda_{i}$.

If we denote by $\boldsymbol{P}^{(1)}$ the matrix whose entries are $\tilde{p}_{i, j}^{(1)}$ then the second identity in Lemma 3 can be written in matrix form as

$$
\boldsymbol{P}^{(1)}=\boldsymbol{A}+\operatorname{diag}[\boldsymbol{d}] \mathscr{B}(\boldsymbol{A} \mid \boldsymbol{d}) .
$$


The $n=3$ case. Now consider the process $\left(X_{t}, Y_{t}\right) \mid \boldsymbol{B}_{3}$. The holding times are exponentially distributed and their parameters are given by $\lambda_{1}, \lambda_{2}, \ldots, \lambda_{k}$ for the $k$ phases at each of the three $(0,1,2)$ levels. Introduce the $2 k \times 2 k$ matrix

$$
\boldsymbol{B}_{3}^{(1)}=\left[\begin{array}{cc}
\boldsymbol{A}^{(1)} & \operatorname{diag}\left[\boldsymbol{d}^{(1)}\right] \\
2 \operatorname{diag}[\boldsymbol{d}] & \boldsymbol{A}
\end{array}\right],
$$

where the entries of the matrix $\boldsymbol{A}^{(1)}$ and the vector $\boldsymbol{d}^{(1)}$ are given by

$$
a_{i, j}^{(1)}=\frac{a_{i, j}}{1-d_{i}} \quad \text { and } \quad d_{i}^{(1)}=\frac{d_{i}}{1-d_{i}} .
$$

Of course, $\boldsymbol{B}_{3}^{(1)}$ is the one-step transition probability matrix of the discrete-time Markov chain $\left(\hat{X}_{t}, \hat{Y}_{t}\right) \mid \boldsymbol{B}_{3}^{(1)}$, derived from $\left(\hat{X}_{t}, \hat{Y}_{t}\right) \mid \boldsymbol{B}_{3}$ through conditioning on staying in levels 1 and 2 . The corresponding continuous-time Markov chain is denoted by $\left(X_{t}, Y_{t}\right) \mid \boldsymbol{B}_{3}^{(1)}$. Analogously to the preceding case, denote by $\left(\tilde{X}_{t}, \tilde{Y}_{t}\right) \mid \boldsymbol{B}_{3}^{(1)}$ the semi-Markov process derived from $\left(X_{t}, Y_{t}\right) \mid \boldsymbol{B}_{3}^{(1)}$ through state space restriction of the latter to level 1 (the first $k$ states of $\left.\left(X_{t}, Y_{t}\right) \mid \boldsymbol{B}_{3}^{(1)}\right)$. In view of Lemma 1 we obtain the following expressions for the LT of the holding time $v_{i, j}^{(1)}$ (the reader should not get confused with the same notation for an analogous holding time in the preceding case) and transition probabilities of this semi-Markov process:

$$
\begin{gathered}
\mathcal{L}\left[v_{i, j}^{(1)}\right](s)=\frac{\lambda_{i}}{\lambda_{i}+s}\left(\frac{a_{i, j}^{(1)}+d_{i}^{(1)} \beta_{i, j}(\boldsymbol{A} \mid 2 \boldsymbol{d}) g_{\boldsymbol{A} \mid 2 \boldsymbol{d}, i, j}\left(\boldsymbol{\Lambda}(s), \lambda_{j} /\left(\lambda_{j}+s\right)\right)}{a_{i, j}^{(1)}+d_{i}^{(1)} \beta_{i, j}(\boldsymbol{A} \mid 2 \boldsymbol{d})}\right), \\
\tilde{p}_{i, j}^{(1)}=a_{i, j}^{(1)}+d_{i}^{(1)} \beta_{i, j}(\boldsymbol{A} \mid 2 \boldsymbol{d}), \quad i, j=1,2, \ldots, k
\end{gathered}
$$

where the matrix $\boldsymbol{\Lambda}(s)$ has been introduced in Lemma 3. The second identity can be written in matrix form as follows:

$$
\boldsymbol{P}^{(1)}=\boldsymbol{A}^{(1)}+\operatorname{diag}\left[\boldsymbol{d}^{(1)}\right] \mathscr{B}(\boldsymbol{A} \mid 2 \boldsymbol{d}) .
$$

Introduce a $k \times k$ matrix $\boldsymbol{P}^{(2)}$, whose $(i, j)$ th entry is given by $\left(1-d_{i}\right) \tilde{p}_{i, j}^{(1)}$, that is,

$$
\mathscr{P}^{(2)}=\operatorname{diag}[\mathbf{1}-\boldsymbol{d}] \boldsymbol{P}^{(1)} \text {. }
$$

Let

$$
\boldsymbol{B}_{3}^{(2)}=\left[\begin{array}{cc}
\boldsymbol{A} & 2 \operatorname{diag}[\boldsymbol{d}] \\
\operatorname{diag}[\boldsymbol{d}] & \mathscr{P}^{(2)}
\end{array}\right] .
$$

It follows from the above construction that $\boldsymbol{B}_{3}^{(2)}$ is the one-step transition probability matrix of the embedded discrete-time Markov chain of the semi-Markov process derived through state space restriction of the continuous-time Markov chain $\left(X_{t}, Y_{t}\right) \mid \boldsymbol{B}_{3}$ to the first two $(0$ and 1$)$ levels. Denote this semi-Markov process by $\left(X_{t}, Y_{t}\right) \mid \boldsymbol{B}_{3}^{(2)}$. Of course, the LTs of the holding times for this semi-Markov process are given by (i) $\lambda_{i} /\left(\lambda_{i}+s\right)$ for a transition from phase $i$ in level 0 to any other phase in that level as well as for a transition from phase $i$ in level 0 or 1 to phase $i$ in level 1 or, respectively, level 0 , and (ii) $v_{i, j}^{(1)}$ (given in Lemma 2) for a transition from phase $i$ in level 1 to phase $j$ in level 1. Furthermore, if we restrict the state space to the states in level 0 (the first $k$ states of $\left(X_{t}, Y_{t}\right) \mid \boldsymbol{B}_{3}^{(2)}$ ), we obtain another semi-Markov process, $\tilde{Y}_{t} \mid \boldsymbol{B}_{3}^{(2)}$ say (here $\tilde{X}_{t}$ can be ignored because it equals 0 ), whose embedded discrete-time Markov chain has $k$ states and the corresponding one-step transition probabilities and holding time distributions are found in the following lemma, which is again a paraphrase of Lemma 1. 
Lemma 4. For the holding times $v_{i, j}^{(2)}, i, j=1, \ldots, k$, of the semi-Markov process $\tilde{Y}_{t} \mid \boldsymbol{B}_{3}^{(2)}$ and the corresponding one-step transition probabilities, we have

$$
\begin{aligned}
& \mathcal{L}\left[v_{i, j}^{(2)}\right](s)=\frac{\lambda_{i}}{\lambda_{i}+s}\left(\frac{a_{i, j}+2 d_{i} \beta_{i, j}\left(\mathcal{P}^{(2)} \mid \boldsymbol{d}\right) g_{\boldsymbol{P}^{(2)} \mid \boldsymbol{d}, i, j}\left(\mathcal{N}^{(1)}(s), \lambda_{j} /\left(\lambda_{j}+s\right)\right)}{a_{i, j}+2 d_{i} \beta_{i, j}\left(\boldsymbol{P}^{(2)} \mid \boldsymbol{d}\right)}\right), \\
& \tilde{p}_{i, j}^{(2)}=a_{i, j}+2 d_{i} \beta_{i, j}\left(\mathcal{P}^{(2)} \mid \boldsymbol{d}\right), \quad i, j=1,2, \ldots, k,
\end{aligned}
$$

where the $(n, m)$ th entry of the $k \times k$ matrix $\mathcal{N}^{(1)}(s)$ is $\mathcal{L}\left[v_{n, m}^{(1)}\right](s)$, which is given by Lemma 3 .

If we denote by $\boldsymbol{P}^{(2)}$ the matrix whose entries are $\tilde{p}_{i, j}^{(2)}$ then the second identity in Lemma 4 can be expressed in matrix form as

$$
\boldsymbol{P}^{(2)}=\boldsymbol{A}+\operatorname{diag}[2 \boldsymbol{d}] \mathscr{B}\left(\boldsymbol{P}^{(2)} \mid \boldsymbol{d}\right) .
$$

Remark 1. Note that from the construction above the semi-Markov process $\tilde{Y}_{t} \mid \boldsymbol{B}_{3}^{(2)}$ is equal to the semi-Markov process which is derived through state space restriction of the continuoustime Markov chain $\left(X_{t}, Y_{t}\right) \mid \boldsymbol{B}_{3}$ to the states in level 0 .

The general case. Suppose that $n \geq 2$ is fixed and consider the matrix $\boldsymbol{B}_{n}$ introduced in (1). For $r=1,2, \ldots, n-1$, let $\boldsymbol{A}^{(r)}$ and $\boldsymbol{d}^{(r)}$ be the matrix and column vector with elements

$$
a_{i, j}^{(r)}=\frac{a_{i, j}}{1-(n-1-r) d_{i}} \quad \text { and } \quad d_{i}^{(r)}=\frac{r d_{i}}{1-(n-1-r) d_{i}} .
$$

In matrix notation we have

$$
\begin{aligned}
& \boldsymbol{A}^{(r)}=(\boldsymbol{I}-(n-1-r) \operatorname{diag}[\boldsymbol{d}])^{-1} \boldsymbol{A}, \\
& \boldsymbol{d}^{(r)}=r(\boldsymbol{I}-(n-1-r) \operatorname{diag}[\boldsymbol{d}])^{-1} \boldsymbol{d} .
\end{aligned}
$$

Note that $\boldsymbol{A}^{(n-1)}=\boldsymbol{A}$ and $\boldsymbol{d}^{(n-1)}=(n-1) \boldsymbol{d}$. Introduce the $k \times k$ matrices $\boldsymbol{P}^{(r)}, \boldsymbol{P}^{(r)}$, and $\boldsymbol{B}^{(r)}$, and, for $i, j=1,2, \ldots, k$, the $\operatorname{LTs} \mu_{i, j}^{(r)}(s)$ and $g_{i, j}^{(r)}(s)$ through the following recurrences:

$$
\begin{aligned}
\mathcal{P}^{(1)} & =\boldsymbol{A}, \\
\boldsymbol{B}^{(1)} & =\boldsymbol{B}\left(\boldsymbol{P}^{(1)} \mid(n-1) \boldsymbol{d}\right), \\
\boldsymbol{P}^{(1)} & =\boldsymbol{A}^{(1)}+\operatorname{diag}\left[\boldsymbol{d}^{(1)}\right] \boldsymbol{B}^{(1)}, \\
\mu_{i, j}^{(0)}(s) & =\frac{\lambda_{i}}{\lambda_{i}+s}, \\
g_{i, j}^{(1)}(s) & =g_{\mathcal{P}^{(1)} \mid(n-1) \boldsymbol{d}, i, j}\left(\boldsymbol{A M}^{(0)}(s), \frac{\lambda_{j}}{\lambda_{j}+s}\right),
\end{aligned}
$$

where the $(i, j)$ th entry of the matrix $\mathcal{M M}^{(0)}(s)$ is $\mu_{i, j}^{(0)}(s)$; and, for $r=2,3, \ldots, n-1$,

$$
\begin{aligned}
\mathcal{P}^{(r)} & =(\boldsymbol{I}-(n-r) \operatorname{diag}[\boldsymbol{d}]) \boldsymbol{P}^{(r-1)}, \\
\boldsymbol{B}^{(r)} & =\boldsymbol{B}\left(\boldsymbol{P}^{(r)} \mid(n-r) \boldsymbol{d}\right), \\
\boldsymbol{P}^{(r)} & =\boldsymbol{A}^{(r)}+\operatorname{diag}\left[\boldsymbol{d}^{(r)}\right] \boldsymbol{B}^{(r)},
\end{aligned}
$$




$$
\begin{aligned}
\mu_{i, j}^{(r-1)}(s) & =\frac{\mu_{i, j}^{(0)}(s)\left(a_{i, j}+d_{i}^{(r-1)} \boldsymbol{B}^{(r-1)}(i, j) g_{i, j}^{(r-1)}(s)\right)}{\boldsymbol{P}^{(r-1)}(i, j)} \\
g_{i, j}^{(r)}(s) & =g_{\mathcal{P}^{(r)} \mid(n-r) \boldsymbol{d}, i, j}\left(\boldsymbol{M}^{(r-1)}(s), \frac{\lambda_{j}}{\lambda_{j}+s}\right) \\
\mu_{i, j}^{(n-1)}(s) & =\frac{\mu_{i, j}^{(0)}(s)\left(a_{i, j}+d_{i}^{(n-1)} \boldsymbol{B}^{(n-1)}(i, j) g_{i, j}^{(n-1)}(s)\right)}{\boldsymbol{P}^{(n-1)}(i, j)}
\end{aligned}
$$

where the $(i, j)$ th entry of the matrix $\boldsymbol{C M}^{(r)}(s)$ is $\mu_{i, j}^{(r)}(s)$ and recalling that the $k \times k$ matrix $\mathscr{B}\left(\mathscr{P}^{(j)} \mid(n-j) \boldsymbol{d}\right)$ is the matrix of absorption probabilities to the last $k$ states of a discrete-time Markov chain whose one-step transition probability matrix is the $2 k \times 2 k$ matrix $\mathscr{P}^{(j)} \mid(n-j) d$.

Theorem 1. Consider the continuous-time Markov chain $\left(X_{t}, Y_{t}\right) \mid \boldsymbol{B}_{n}$ introduced in Section 2. The parameters of the exponentially distributed holding times are given by $\lambda_{i}$ for phase $i$ at each level. Denote by $\tilde{\tilde{Y}}_{t}$ the semi-Markov process derived from $\left(X_{t}, Y_{t}\right) \mid \boldsymbol{B}_{n}$ by state space restriction to the states in level 0 . Then the one-step transition probability matrix of the embedded discrete-time Markov chain (with $k$ states) of $\tilde{\tilde{Y}}_{t}$ is given by $\boldsymbol{P}^{(n-1)}$ and the Laplace transform of the corresponding holding time distribution at state $i$, given that the next state to be visited is $j$, is given by $\mu_{i, j}^{(n-1)}$, where $\boldsymbol{P}^{(n-1)}$ and $\mu_{i, j}^{(n-1)}$ are found using recurrences (13)-(18), with initialisation given by (8)-(12).

Proof. The cases in which $n=2$ and $n=3$ follow from Lemmas 3 and 4, respectively. For a general $n$, we make step-by-step state space restrictions, reducing the state space by one level at a time starting from the last level. The derivation of the relevant holding time distributions follows the idea used in the preceding special cases. Therefore, we confine ourselves to a brief statement of the necessary steps and leave the technical details to the reader. In the first step we restrict the state space of $\left(X_{t}, Y_{t}\right) \mid \boldsymbol{B}_{n}$ to the first $n-1$ levels and obtain a semi-Markov process, $\left(X_{1}^{(1)}, Y_{t}^{(1)}\right) \mid \boldsymbol{B}_{n}$ say, with $(n-1) k$ states. The one-step transition probability matrix of its embedded discrete-time Markov chain is found to be equal to the matrix derived from $\boldsymbol{B}_{n}$ by deleting its last row and last column and then replacing the bottom-right matrix $\boldsymbol{A}$ by $\boldsymbol{P}^{(2)}$. Also, the holding time distributions of $\left(X_{t}^{(1)}, Y_{t}^{(1)}\right) \mid \boldsymbol{B}_{n}$ are the same as those for the same states of the continuous-time Markov chain $\left(X_{t}, Y_{t}\right) \mid \boldsymbol{B}_{n}$, except for those associated with transitions between the phases in the last level (level $n-2)$ whose LTs are found to be equal to the $\mu_{i, j}^{(1)}(s)$. In the next step we restrict the state space of $\left(X_{1}^{(1)}, Y_{t}^{(1)}\right) \mid \boldsymbol{B}_{n}$ to the first $n-2$ levels and obtain a semi-Markov process, $\left(X_{1}^{(2)}, Y_{t}^{(2)}\right) \mid \boldsymbol{B}_{n}$ say, with $(n-2) k$ states. The one-step transition probability matrix of its embedded discrete-time Markov chain is found to be equal to the matrix derived from $\boldsymbol{B}_{n}$ by deleting its last two rows and last two columns and then replacing the bottom-right matrix $\boldsymbol{A}$ by $\boldsymbol{P}^{(3)}$. Also, the holding time distributions of $\left(X_{t}^{(2)}, Y_{t}^{(2)}\right) \mid \boldsymbol{B}_{n}$ are the same as those for the same states of the continuous-time Markov chain $\left(X_{t}, Y_{t}\right) \mid \boldsymbol{B}_{n}$, except for those associated with transitions between the phases in the last level (level $n-3$ ) whose LTs are found to be equal to $\mu_{i, j}^{(2)}(s)$. By induction we obtain a semi-Markov process $\left(X_{t}^{(n-1)}, Y_{t}^{(n-1)}\right) \mid \boldsymbol{B}_{n}$ with $k$ states, whose holding times have LTs given by $\mu_{i, j}^{(n-1)}$ and whose corresponding one-step transition probability matrix is given by $\boldsymbol{P}^{(n-1)}$. From the construction of the semi-Markov processes $\underset{\tilde{Y}}{(}\left(X_{t}^{(r)}, Y_{t}^{(r)}\right) \mid \boldsymbol{B}_{n}$, it is clear that $\left(X_{t}^{(n-1)}, Y_{t}^{(n-1)}\right) \mid \boldsymbol{B}_{n}$ is equal to the semi-Markov process $\tilde{Y}_{t} \mid \boldsymbol{B}_{n}$. Thus, the statement of Theorem 1 holds.

Consider again the continuous-time Markov chain $\left(X_{t}, Y_{t}\right) \mid \boldsymbol{B}_{n}$. Denote by $\delta_{i, j}^{(r)}(s), r=$ $1,2, \ldots, n-1$, the LT of the waiting time to move from phase $i$ in level $r$ to the set of states 
in level 0 , given that the reached phase in level 0 is $j$, and denote by $q_{i, j}^{(r)}$ the corresponding probability that level 0 is reached at phase $j$. For $r=1,2, \ldots, n-1$, let $\boldsymbol{Q}^{(r)}$ denote the $k \times k$ matrix whose $(i, j)$ th entry is $q_{i, j}^{(r)}$. The following theorem is a by-product of the method used to derive Theorem 1 .

Theorem 2. The following recurrences hold for $\delta_{i, j}^{(r)}(s)$ and $\boldsymbol{Q}^{(r)}, i, j=1,2, \ldots, k$ :

$$
\begin{aligned}
\delta_{i, j}^{(1)}(s) & =g_{i, j}^{(n-1)}(s), \\
\boldsymbol{Q}^{(1)} & =\boldsymbol{B}^{(n-1)}, \\
\delta_{i, j}^{(r)}(s) & =\sum_{m=1}^{k} g_{i, m}^{(n-r)}(s) \boldsymbol{B}^{(n-r)}(i, m) \delta_{m, j}^{(r-1)}(s), \\
\boldsymbol{Q}^{(r)} & =\boldsymbol{B}^{(n-r)} \boldsymbol{Q}^{(r-1)}, \quad r=2,3, \ldots, n-1,
\end{aligned}
$$

where $\boldsymbol{B}^{(r)}$ and $g_{i, j}^{(r)}(s)$ are given above.

Proof. Note the meaning of $g_{i, j}^{(r)}(s), r=1,2, \ldots, n-1$. Actually, $g_{i, j}^{(r)}(s)$ is the LT of the excursion from phase $i$ in level $n-r$ before entering level $n-r-1$, given that level is entered via phase $j$. The probability that, after that excursion, the level $n-r-1$ is reached via phase $j$ is given by the $(i, j)$ th entry of matrix $\boldsymbol{B}^{(r)}$. In other words, if $r=n-1$, we have

$$
\delta_{i, j}^{(1)}(s)=g_{i, j}^{(n-1)}(s) .
$$

Also, the probability that level 0 has been reached via phase $j$ is given by the $(i, j)$ th entry of matrix $\mathscr{B}^{(n-1)}$. Furthermore, note that the LT of the waiting time to move from phase $i$ in level 2 to level 1, given that the latter is reached via phase $j$, is given by

$$
\delta_{i, j}^{(2)}(s)=\sum_{m=1}^{k} g_{i, m}^{(n-2)}(s) \boldsymbol{B}^{(n-2)}(i, m) \delta_{m, j}^{(1)}(s)
$$

recall that $\boldsymbol{B}^{(n-2)}(i, m)$ is the $(i, m)$ th entry of the matrix $\boldsymbol{B}^{(n-2)}$. Also, the probability that level 1 has been reached via phase $j$ is equal to

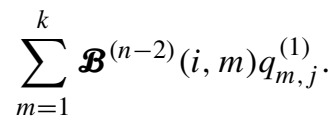

By induction we obtain recurrences (19)-(22).

\subsection{Reward processes}

In this subsection we derive explicit, closed-form expressions for reward functions associated with visits to, and sojourns in, the states in level 0 of the two-dimensional process $\left(X_{t}, Y_{t}\right) \mid \boldsymbol{B}_{n}$, which has been introduced above. In order to keep the expressions neater and consistent with related expressions on reward functions for semi-Markov processes, we convert our twodimensional processes to one-dimesional processes. More specifically, define a semi-Markov process $V_{t}$ by $V_{t}=Y_{t}+k X_{t}$, where $X_{t}$ and $Y_{t}$ are the components of the two-dimensional process $\left(X_{t}, Y_{t}\right) \mid \boldsymbol{B}_{n}$. Then $V_{t}$ has $k n$ states. Denote the initial probabilities by $p_{i, j}, i=$ $0,1, \ldots, n-1, j=1,2, \ldots, k$, where $p_{i, j}$ is the initial probability for state $j+i k$. The 
quantity of interest is a reward function associated with the sojourns in the first $k$ states and visits to them. More specifically, introduce the reward function

$$
H(t)=\sum_{i=1}^{2 k} \sum_{j=1}^{k} h_{i, j} N_{i, j}(t)+\sum_{i=1}^{k} h_{i} S_{i}(t)+\sum_{i=1}^{k} h_{i}^{\star} \mathbf{1}_{\left\{V_{0}=i\right\}}
$$

where $\mathbf{1}_{\{\cdot\}}$ is an indicator function which is equal to 1 if the event $\{\cdot\}$ occurs and 0 otherwise, $S_{i}(t)$ is the time spent in state $i$ by $V_{t}$, and $N_{i, j}(t)$ is the number of transitions from state $i$ to state $j$ of $V_{t}$ within the time interval $[0, t]$.

Now consider the semi-Markov process $\left(X_{t}^{(n-2)}, Y_{t}^{(n-2)}\right) \mid \boldsymbol{B}_{n}$ introduced in the proof of Theorem 1. Convert it into a one-dimensional process via $V_{t}^{(n-2)}=Y_{t}^{(n-2)}+k X_{t}^{(n-2)}$. Then $V_{t}^{(n-2)}$ has $2 k$ states and the one-step transition probability matrix of its embedded discrete-time Markov chain is

$$
\boldsymbol{P}_{V^{(n-2)}}=\left[\begin{array}{cc}
\boldsymbol{A} & (n-1) \operatorname{diag}[\boldsymbol{d}] \\
\operatorname{diag}[\boldsymbol{d}] & \boldsymbol{P}^{(n-2)}
\end{array}\right] .
$$

Recall that, for $i, j=1,2, \ldots, k$, the LTs of the holding time distributions are given by the $\lambda_{i} /\left(s+\lambda_{i}\right)$ for pairs of states $(i, j)$ or $(i, i+k)$ or $(i+k, i)$ and by $\mu_{i, j}^{(n-2)}$ for pairs of states $(i+k, j+k)$.

Introduce the following reward function associated with the process $V_{t}^{(n-2)}$ :

$$
\tilde{H}(t)=\sum_{i=1}^{2 k} \sum_{j=1}^{k} h_{i, j} \tilde{N}_{i, j}(t)+\sum_{i=1}^{k} h_{i} \tilde{S}_{i}(t)+\sum_{i=1}^{k} h_{i}^{\star} \mathbf{1}_{\left\{V_{0}^{(n-2)}=i\right\}},
$$

where $\tilde{S}_{i}(t)$ is the time spent in state $i$ by the semi-Markov process $V_{t}^{(n-2)}, \tilde{N}_{i, j}(t)$ is the number of transitions from state $i$ to state $j$ of $V_{t}^{(n-2)}$ within the time interval $[0, t]$, and $h_{i, j}, h_{i}$, and $h_{i}^{\star}$ are the quantities associated with the reward function $H(t)$ introduced in (23). It is clear that, for each $i, i=1,2, \ldots, k$ (the first $k$ states of the process $V_{t}^{(n-2)}$ ),

$$
\mathrm{P}\left(H(t) \leq x \mid V_{0}=i\right)=\mathrm{P}\left(\tilde{H}(t) \leq x \mid V_{0}^{(n-2)}=i\right)
$$

Denote by $\tau_{\left(i_{1}, j_{1}\right),\left(i_{2}, j_{2}\right)}$ the waiting time to move from state $i_{1}$ to state $i_{2}$, given that the next state visited after $i_{1}$ is state $j_{1}$ and that the next state visited after state $i_{2}$ is state $j_{2}$, in the semi-Markov process $V_{t}^{(n-2)}$. Note that there is a random number of visits to state $i_{2}$ before $\tau_{\left(i_{1}, j_{1}\right),\left(i_{2}, j_{2}\right)}$; these are visits to $i_{2}$ which are succeeded by jumps to states different than state $j_{2}$. The waiting times $\tau_{(i, i),(i, i)}$ and $\tau_{(i, j),(i, j)}$ (which are strictly positive) are the first return times to state $i$, under the relevant conditions. Denote by $D_{\left(i_{1}, j_{1}\right),\left(i_{2}, j_{2}\right)}$ the joint (two-dimensional) distribution of $\tau_{\left(i_{1}, j_{1}\right),\left(i_{2}, j_{2}\right)}$ and $\tilde{H}\left(\tau_{\left(i_{1}, j_{1}\right),\left(i_{2}, j_{2}\right)}\right)$, that is, the joint distribution of that waiting time with the associated reward $\tilde{H}$ accumulated within that waiting time. Denote the Laplace transform

$$
\int_{0}^{\infty} \int_{0}^{\infty} \exp \left\{-s_{1} t-s_{2} x\right\} \mathrm{P}\left(\tilde{H}(t) \leq x \mid V_{0}^{(n-2)}=i\right) \mathrm{d} t \mathrm{~d} x
$$

by $\mathcal{L}\left[\mathrm{P}\left(\tilde{H}(t) \leq x \mid V_{0}^{(n-2)}=i\right)\right]\left(s_{1}, s_{2}\right)$. Then from Theorem 4 in Appendix A we obtain, 
for $r_{1}=1,2, \ldots, k$ (the factor $\exp \left\{-s_{2} h_{r_{1}}^{\star}\right\}$ is due to the reward associated with the initial state $\left.r_{1}\right)$,

$$
\begin{aligned}
\mathscr{L}\left[\mathrm{P}\left(\tilde{H}(t) \leq x \mid V_{0}^{(n-2)}=r_{1}\right)\right]\left(s_{1}, s_{2}\right) \\
=\exp \left\{-s_{2} h_{r_{1}}^{\star}\right\}\left(\sum_{r_{2}, i, j=1}^{2 k} \frac{\left(1-\mu_{i, j}\left(s_{1}+s_{2} h_{i}\right)\right) \mathcal{L}\left[D_{\left(r_{1}, r_{2}\right),(i, j)}\right]\left(s_{1}, s_{2}\right)}{s_{2}\left(s_{1}+s_{2} h_{i}\right)\left(1-\mathcal{L}\left[D_{(i, j),(i, j)}\right]\left(s_{1}, s_{2}\right)\right)}\right. \\
\quad \times \boldsymbol{P}_{V^{(n-2)}}\left(r_{1}, r_{2}\right) \boldsymbol{P}_{V^{(n-2)}}(i, j) \\
\left.+\sum_{r_{2}=1}^{2 k} \frac{\left(1-\mu_{r_{1}, r_{2}}\left(s_{1}+s_{2} h_{r_{1}}\right)\right)}{s_{2}\left(s_{1}+s_{2} h_{r_{1}}\right)} \boldsymbol{P}_{V^{(n-2)}}\left(r_{1}, r_{2}\right)\right)
\end{aligned}
$$

where the $\mu_{i, j}$ are the LTs of the holding time distributions of the semi-Markov process $V_{t}^{(n-2)}$. Now consider

$$
\mathrm{P}\left(H(t) \leq x \mid V_{0}=u+r k\right), \quad u=1,2, \ldots, k, r=1,2, \ldots, n-1 .
$$

Fix $u$ and $r$, and augment the state space of the semi-Markov process $V_{t}^{(n-2)}$ by one state, 0 say, which will play the role of the initial state. The nonzero one-step transition probabilities from state 0 are only to states 1 through $k$ and they are equal to $q_{u, j}^{(r)}$ for $j=1,2, \ldots, k$. Also, the associated holding times with such transitions are given by $\delta_{u, j}^{(r)}$. Recall that the quantities $q_{u, j}^{(r)}$ and $\delta_{u, j}^{(r)}$ are introduced in Theorem 2. Denote this new semi-Markov process with $2 k+1$ states by $V_{t}^{(u, r)}$ and consider the reward function

$$
\tilde{\tilde{H}}(t)=\sum_{i=1}^{2 k} \sum_{j=1}^{k} h_{i, j} \tilde{\tilde{N}}_{i, j}(t)+\sum_{i=1}^{k} h_{i} \tilde{\tilde{S}}_{i}(t)+\sum_{i=1}^{k} h_{i}^{\star} \tilde{\tilde{N}}_{0, i}(t),
$$

where $\tilde{\tilde{S}}_{i}(t)$ and $\tilde{\tilde{N}}_{i, j}(t)$ are the relevant quantities associated with the augmented process $V_{t}^{(u, r)}$. From the construction of $V_{t}^{(u, r)}$, it is clear that

$$
\mathrm{P}\left(H(t) \leq x \mid V_{0}=u+r k\right)=\mathrm{P}\left(\tilde{\tilde{H}}(t) \leq x \mid V_{0}^{(u, r)}=0\right) .
$$

Therefore, again from Theorem 4 we obtain (recall that there is no reward associated with sojourns in state 0 , so $h_{0}=0$ )

$$
\begin{aligned}
& \mathcal{L}\left[\mathrm{P}\left(\tilde{\tilde{H}}(t) \leq x \mid Y_{0}^{(u, r)}=0\right)\right]\left(s_{1}, s_{2}\right) \\
& =\sum_{r_{2}=1}^{k} \sum_{i, j=1}^{2 k} \frac{\left(1-\mu_{i, j}\left(s_{1}+s_{2} h_{i}\right)\right) \mathcal{L}\left[D_{\left(0, r_{2}\right),(i, j)}^{(u, r)}\right]\left(s_{1}, s_{2}\right)}{s_{2}\left(s_{1}+s_{2} h_{i}\right)\left(1-\mathcal{L}\left[D_{(i, j),(i, j)}\right]\left(s_{1}, s_{2}\right)\right)} q_{u, r_{2}}^{(r)} \boldsymbol{P}_{V^{(n-2)}}(i, j) \\
& \quad+\sum_{r_{2}=1}^{k} \frac{\left(1-\delta_{u, r_{2}}^{(r)}\left(s_{1}\right)\right)}{s_{2} s_{1}} q_{u, r_{2}}^{(r)},
\end{aligned}
$$

where the $\mu_{i, j}$ are the LTs of the holding time distributions of the semi-Markov process $V_{t}^{(n-2)}$ and $D_{\left(0, r_{2}\right),(i, j)}^{(u, r)}$ is the joint distribution of the waiting time $\tau_{\left(0, r_{2}\right),(i, j)}$ (for the semi-Markov process $V_{t}^{(u, r)}$ ) together with the associated reward accumulated during that waiting time.

In view of (24), (25), and (26), we arrive at the following theorem. 
Theorem 3. For the Laplace transform $\int_{0}^{\infty} \int_{0}^{\infty} \exp \left\{-s_{1} t-s_{2} x\right\} \mathrm{P}(H(t) \leq x) \mathrm{d} t \mathrm{~d} x$ of the cumulative distribution function of the reward function $H(t)$ given in (23), we have the following expression:

$$
\begin{aligned}
\sum_{r_{1}=1}^{k} p_{0, r_{1}} \mathrm{e}^{-s_{2} h_{r_{1}}^{\star}}\left(\sum_{i, j, r_{2}=1}^{2 k} \frac{\left(1-\mu_{i, j}\left(s_{1}+s_{2} h_{i}\right)\right) \mathcal{L}\left[D_{\left(r_{1}, r_{2}\right),(i, j)}\right]\left(s_{1}, s_{2}\right)}{s_{2}\left(s_{1}+s_{2} h_{i}\right)\left(1-\mathcal{L}\left[D_{(i, j),(i, j)}\right]\left(s_{1}, s_{2}\right)\right)}\right. \\
\quad \times \boldsymbol{P}_{V^{(n-2)}}\left(r_{1}, r_{2}\right) \boldsymbol{P}_{V^{(n-2)}}(i, j) \\
+\sum_{r_{2}=1}^{2 k} \frac{1-\mu_{r_{1}, r_{2}}\left(s_{1}+s_{2} h_{r_{1}}\right)}{s_{2}\left(s_{1}+s_{2} h_{r_{1}}\right)} \boldsymbol{P}_{\left.V^{(n-2)}\left(r_{1}, r_{2}\right)\right)} \\
+\sum_{r=1}^{n-1} \sum_{u=1}^{k} p_{u, r}\left(\sum_{r_{2}=1}^{k} \sum_{i, j=1}^{2 k} \frac{\left(1-\mu_{i, j}\left(s_{1}+s_{2} h_{i}\right)\right) \mathcal{L}\left[D_{\left(0, r_{2}\right),(i, j)}^{(u, r)}\right]\left(s_{1}, s_{2}\right)}{s_{2}\left(s_{1}+s_{2} h_{i}\right)\left(1-\mathcal{L}\left[D_{(i, j),(i, j)}\right]\left(s_{1}, s_{2}\right)\right)} q_{u, r_{2}}^{(r)} \boldsymbol{P}_{\left.V^{(n-2)}\right)}(i, j)\right. \\
\left.+\sum_{r_{2}=1}^{k} \frac{\left(1-\delta_{u, r_{2}}^{(r)}\left(s_{1}\right)\right)}{s_{2} s_{1}} q_{u, r_{2}}^{(r)}\right),
\end{aligned}
$$

where the $\mu_{i, j}$ are the LTs of the holding time distributions of the semi-Markov process $V_{t}^{(n-2)}$, and the $p_{i, j}$ are the initial probabilities of $V_{t}$, as introduced at the beginning of this subsection.

Explicit, closed-form expressions for the Laplace transforms

$$
\mathcal{L}\left[D_{\left(i_{1}, j_{1}\right),\left(i_{2}, j_{2}\right)}\right] \quad \text { and } \quad \mathcal{L}\left[D_{\left(0, j_{1}\right),\left(i_{2}, j_{2}\right)}^{(u, r)}\right]
$$

can be derived as explained in Appendix A. Recall that the number of states, $2 k+1$, of the embedded Markov chains is small, for example, 9 and 15 for uncle-type and cousin-type relationships, respectively.

\subsection{Implementation}

We summarise below the algorithm for calculating an explicit closed-form expression for the Laplace transform of the cumulative distribution function of the reward $H(t)$ given in (23). All steps are implementable using a computer algebra package such as MAPLE ${ }^{\circledR}$ or MATHEMATICA $^{\circledR}$.

- Using the identities given in (3), evaluate the initial quantities given in (8)-(12), that is, $\boldsymbol{B}\left(\boldsymbol{P}^{(1)} \mid(n-1) \boldsymbol{d}\right), \boldsymbol{P}^{(1)}$, and

$$
g_{i, j}^{(1)}(s)=g_{\boldsymbol{A} \mid(n-1) \boldsymbol{d}, i, j}\left(\boldsymbol{\Lambda}(s), \frac{\lambda_{j}}{\lambda_{j}+s}\right),
$$

where the matrix $\boldsymbol{\Lambda}(s)$ has been introduced in Lemma 3 and recall from the line after (12) that $\boldsymbol{M M}^{(0)}(s)=\boldsymbol{\Lambda}(s)$. Using the recurrences given in (8)-(18), and (19)-(22) (cf. Theorems 1 and 2), evaluate the Laplace transforms $\mu_{i, j}^{(n-2)}$ and $\delta_{i, j}^{(r)}$, and the matrices $\mathcal{P}^{(n-2)}$ and $\boldsymbol{Q}^{(r)}$.

- Derive explicit closed-form expressions for the Laplace transforms $\mathscr{L}\left[D_{\left(i_{1}, j_{1}\right),\left(i_{2}, j_{2}\right)}\right]$ and $\mathcal{L}\left[D_{\left(0, j_{1}\right),\left(i_{2}, j_{2}\right)}^{(u, r)}\right.$, that is, the Laplace transforms of the joint distributions of relevant first passage times together with the associated accumulated rewards. 
- From the quantities evaluated above, calculate an explicit closed-form expression for the Laplace transform of the cumulative distribution function of any relevant reward function $H(t)$ using the result in Theorem 3.

A successful numerical inversion of the Laplace transform yields cumulative probabilities of the reward function $H(t)$. Abate and Whitt (2006) provided several algorithms for such inversions (cf. also Abate et al. (1998) and Choudhury et al. (1994)).

\section{Applications to IBD}

The relevant reward functions for uncle-type and cousin-type relationships are

$$
H_{1}(t)=S_{1}(t)+S_{2}(t), \quad H_{2}(t)=\sum_{i=1}^{2 k}\left(N_{i, 1}(t)+N_{i, 2}(t)\right)+\mathbf{1}_{\left\{V_{0}=1\right\}}+\mathbf{1}_{\left\{V_{0}=2\right\}},
$$

that is, the accumulated sojourn in the first two states and the number of entries to the first two states. Their interpretation in terms of IBD has been explained in Section 2. The explicit expressions for the reward LTs are relatively lengthy and we confine ourselves to listing the ones for the uncle/nephew relationship which can be written on one line. More specifically, for the Laplace transform $\mathcal{L}\left[\mathrm{P}\left(H_{1}(t) \leq x\right)\right]\left(s_{1}, s_{2}\right)$ of the cumulative distribution function of the reward function $H_{1}(t)$ corresponding to the amount of IBD genome shared by an uncle and his nephew on a chromosomal segment of length $t$ morgans, we obtain the following explicit expression:

$\frac{4 s_{1}^{3}+6 s_{1}^{2} s_{2}+2 s_{1} s_{2}^{2}+72 s_{1}^{2}+72 s_{1} s_{2}+13 s_{2}^{2}+416 s_{1}+208 s_{2}+768}{4 s_{2}\left(s_{1}^{4}+2 s_{1}^{3} s_{2}+s_{1}^{2} s_{2}^{2}+18 s_{1}^{3}+27 s_{1}^{2} s_{2}+9 s_{1} s_{2}^{2}+104 s_{1}^{2}+104 s_{1} s_{2}+16 s_{2}^{2}+192 s_{1}+96 s_{2}\right)}$.

For discrete reward functions, such as $\mathrm{H}_{2}(t)$, it is more convenient to use the mixed (generating function - Laplace) transform

$$
\sum_{x=0}^{\infty} \int_{0}^{\infty} x^{s_{2}} \exp \left\{-s_{1} t\right\} \mathrm{P}(H(t) \leq x) \mathrm{d} t
$$

For the mixed transform of the cumulative distribution function of the reward function $\mathrm{H}_{2}(t)$ corresponding to the number of IBD pieces on a chromosomal segment of length $t$ morgans, we obtain the following explicit expression:

$$
\frac{4 s_{1}^{2}+9 s_{1} s_{2}+2 s_{2}^{2}+47 s_{1}+54 s_{2}+136}{-4 \ln \left(s_{2}\right)\left(s_{1}^{3}-4 s_{1} s_{2}^{2}+14 s_{1}^{2}-9 s_{1} s_{2}-28 s_{2}^{2}+61 s_{1}-52 s_{2}+80\right)} .
$$

We also list some calculation results for the uncle/nephew and first-cousin relationships in Tables 1 and 2. These have been calculated using the Gaver-Stehfest algorithm (cf. Abate and Whitt (2006)). From Table 1 we also note that the distribution of the proportion of shared genome for the uncle/nephew relationship is symmetric.

Remark 2. There is a matrix exponential representation for the one-dimensional Laplace transform of the reward function $H_{1}(t)$ (cf. Ball et al. (1994, p. 929)). Effective numerical evaluations of such matrix exponentials are possible if the matrix in the exponential is diagonalizable. Since the transition matrices for uncle-type and cousin-type relationships are diagonalizable, then such a numerical approach for $H_{1}(t)$ is feasible and will be investigated by the authors elsewhere. 
TABLE 1: Cumulative probabilities $F(x)$ for the proportion of the genome shared IBD on a chromosomal segment of length $d$ morgans.

\begin{tabular}{llllllllllll}
\hline$d$ & \multicolumn{10}{c}{$x$} \\
\cline { 2 - 9 } & 0.45 & 0.40 & 0.35 & 0.30 & 0.25 & 0.20 & 0.15 & 0.10 & 0.05 & 0.00 \\
\hline \multicolumn{10}{c}{ Uncle/nephew relationship } \\
\hline 2 & 0.970 & 0.911 & 0.808 & 0.666 & 0.500 & 0.334 & 0.192 & 0.090 & 0.030 & 0.003 \\
3 & 0.990 & 0.952 & 0.859 & 0.702 & 0.500 & 0.298 & 0.141 & 0.048 & 0.010 & 0.0003 \\
\hline \multicolumn{10}{c}{ First-cousin relationship } \\
\hline 2 & 0.999 & 0.995 & 0.982 & 0.953 & 0.895 & 0.784 & 0.645 & 0.451 & 0.242 & 0.064 \\
3 & 0.9999 & 0.999 & 0.994 & 0.978 & 0.932 & 0.832 & 0.657 & 0.417 & 0.174 & 0.019 \\
\hline
\end{tabular}

TABLE 2: Cumulative probabilities $F(k)$ for the number of shared IBD pieces, $k$, on a chromosomal segment of length $d$ morgans.

\begin{tabular}{cccccccccccccc}
\hline$d$ & \multicolumn{10}{c}{$k$} \\
\cline { 2 - 12 } & 0 & 1 & 2 & 3 & 4 & 5 & 6 & 7 & 8 & 9 & 10 & 11 \\
\hline \multicolumn{10}{c}{ Uncle/nephew relationship } \\
\hline 2 & 0.080 & 0.030 & 0.107 & 0.250 & 0.429 & 0.626 & 0.775 & 0.875 & 0.943 & 0.978 & 0.991 & 0.997 \\
3 & 0.001 & 0.004 & 0.018 & 0.058 & 0.131 & 0.261 & 0.411 & 0.558 & 0.702 & 0.821 & 0.898 & 0.946 \\
\hline \multicolumn{110}{c}{ First-cousin relationship } \\
\hline 2 & 0.080 & 0.161 & 0.343 & 0.545 & 0.714 & 0.844 & 0.920 & 0.961 & 0.985 & 0.995 & 0.998 & 0.999 \\
3 & 0.024 & 0.059 & 0.153 & 0.295 & 0.451 & 0.617 & 0.747 & 0.841 & 0.913 & 0.958 & 0.979 & 0.991 \\
\hline
\end{tabular}

\section{Appendix A}

In this appendix we extend a result of Stefanov (2006) on reward functions for semi-Markov processes to the case where the holding time in a given state depends on both the current state and the next state visited. The notation in this appendix is self-contained and local to it. Let $Y_{t}$ be a semi-Markov process with $m$ states, and let the one-step transition probability matrix for the embedded discrete-time Markov chain and its $(i, j)$ th entry be denoted by $\boldsymbol{P}$ and $p_{i, j}$, respectively. The LT of the holding time distribution at state $i$, given that the next state to be visited is state $j$, is denoted by $\mu_{(i, j)}$. Introduce the following reward function:

$$
\tilde{H}(t)=\sum_{i, j=1}^{m} h_{i, j} \tilde{N}_{i, j}(t)+\sum_{i=1}^{m} h_{i} \tilde{S}_{i}(t),
$$

where $\tilde{S}_{i}(t)$ is the time spent in state $i$ by the semi-Markov process $Y_{t}$ and $\tilde{N}_{i, j}(t)$ is the number of transitions from state $i$ to state $j$ of $Y_{t}$ within the time interval $[0, t]$.

Denote by $\tau_{\left(i_{1}, j_{1}\right),\left(i_{2}, j_{2}\right)}$ the waiting time to move from state $i_{1}$ to state $i_{2}$, given that the next state visited after $i_{1}$ is state $j_{1}$ and that the next state visited after state $i_{2}$ is state $j_{2}$, in the semi-Markov process $Y_{t}$. Note that there is a random number of visits to state $i_{2}$ before $\tau_{\left(i_{1}, j_{1}\right),\left(i_{2}, j_{2}\right)}$; these are visits to $i_{2}$ which are succeeded by jumps to states different than state $j_{2}$. 
The waiting times $\tau_{(i, i),(i, i)}$ and $\tau_{(i, j),(i, j)}$ (which are strictly positive) are the first return times to state $i$, under the relevant conditions. Denote by $D_{\left(i_{1}, j_{1}\right),\left(i_{2}, j_{2}\right)}$ the joint (two-dimensional) distribution of $\tau_{\left(i_{1}, j_{1}\right),\left(i_{2}, j_{2}\right)}$ and $\tilde{H}\left(\tau_{\left(i_{1}, j_{1}\right),\left(i_{2}, j_{2}\right)}\right)$, that is, the joint distribution of that waiting time with the associated reward $\tilde{H}$ accumulated within that waiting time.

Theorem 4. For the LT of $\mathrm{P}\left(\tilde{H}(t) \leq x \mid Y_{0}=r_{1}\right)$ with respect to $t$ and $x$, we have

$$
\begin{aligned}
& \mathcal{L}\left[\mathrm{P}\left(\tilde{H}(t) \leq x \mid Y_{0}=r_{1}\right)\right]\left(s_{1}, s_{2}\right) \\
& =\sum_{r_{2}, i, j=1}^{m} \frac{\left(1-\mu_{(i, j)}\left(s_{1}+s_{2} h_{i}\right)\right) \mathcal{L}\left[D_{\left(r_{1}, r_{2}\right),(i, j)}\right]\left(s_{1}, s_{2}\right)}{s_{2}\left(s_{1}+s_{2} h_{i}\right)\left(1-\mathcal{L}\left[D_{(i, j),(i, j)}\right]\left(s_{1}, s_{2}\right)\right)} p_{r_{1}, r_{2}} p_{i, j} \\
& \quad+\sum_{r_{2}=1}^{m} \frac{\left(1-\mu_{\left(r_{1}, r_{2}\right)}\left(s_{1}+s_{2} h_{i}\right)\right)}{s_{2}\left(s_{1}+s_{2} h_{i}\right)} p_{r_{1}, r_{2} .} .
\end{aligned}
$$

Proof. Denote by $w_{t}$ the waiting time starting from time epoch $t$ and ending when a jump in the semi-Markov process $Y_{t}$ occurs. That is, if the process is in state $i$ at time $t$ then at $t+w_{t}$ the process enters the next state visited after state $i$. Also, let

$$
\tau_{(i, j)}^{(k)}=\inf \left\{u>\tau_{(i, j)}^{(k-1)}: Y_{u}=i, Y_{u+w_{u}}=j, \text { and } Y_{t} \text { jumps in }\left(\tau_{(i, j)}^{(k-1)}, u\right]\right\}, \quad k=1,2, \ldots,
$$

where $\tau_{(i, j)}^{(0)}=0$; recall that a semi-Markov process may jump without changing state. Of course,

$$
\mathrm{P}\left(\tilde{H}(t) \leq x \mid Y_{0}=r_{1}\right)=\sum_{r_{2}, i, j=1}^{m} \mathrm{P}\left(\tilde{H}(t) \leq x, Y_{w_{0}}=r_{2}, Y_{t}=i, Y_{t+w_{t}}=j \mid Y_{0}=r_{1}\right) .
$$

Case 1: $(i, j) \neq\left(r_{1}, r_{2}\right)$. First note that

$$
\begin{aligned}
& \mathrm{P}\left(\tilde{H}(t) \leq x, Y_{w_{0}}=r_{2}, Y_{t}=i, Y_{t+w_{t}}=j \mid Y_{0}=r_{1}\right) \\
& \quad=\mathrm{P}\left(\tilde{H}(t) \leq x, Y_{t}=i \mid Y_{0}=r_{1}, Y_{w_{0}}=r_{2}, Y_{t+w_{t}}=j\right) p_{r_{1}, r_{2}} p_{i, j} .
\end{aligned}
$$

Furthermore,

$$
\begin{aligned}
& \mathrm{P}\left(\tilde{H}(t) \leq x, Y_{t}=i \mid Y_{0}=r_{1}, Y_{w_{0}}=r_{2}, Y_{t+w_{t}}=j\right) \\
& \quad=\sum_{k=1}^{\infty} \mathrm{P}\left(\tilde{H}(t) \leq x, Y_{t}=i, \tau_{(i, j)}^{(k)} \leq t<\tau_{(i, j)}^{(k+1)} \mid Y_{0}=r_{1}, Y_{w_{0}}=r_{2}, Y_{t+w_{t}}=j\right) .
\end{aligned}
$$

From the basic properties of semi-Markov processes, it follows that, for a given pair of states $(i, j)$, the process regenerates at each $\tau_{(i, j)}^{(k)}$. Therefore, using the same arguments as those applied in the proof of Theorem 2.1 of Stefanov (2006) for deriving the LT of a similar conditional probability, for the LT of

$$
\mathrm{P}\left(\tilde{H}(t) \leq x, Y_{t}=i, \tau_{(i, j)}^{(k)} \leq t<\tau_{(i, j)}^{(k+1)} \mid Y_{0}=r_{1}, Y_{w_{0}}=r_{2}, Y_{t+w_{t}}=j\right),
$$

we obtain the expression

$$
\frac{\left.\left(1-\mu_{(i, j)}\left(s_{1}+s_{2} h_{i}\right)\right) \mathcal{L}\left[D_{\left(r_{1}, r_{2}\right),(i, j)}\right]\left(s_{1}, s_{2}\right)\left(\mathcal{L}\left[D_{(i, j),(i, j)}\right]\left(s_{1}, s_{2}\right)\right)\right)^{k-1}}{s_{2}\left(s_{1}+s_{2} h_{i}\right)} .
$$


In view of (27), (28), and (29), for the LT of

$$
\mathrm{P}\left(\tilde{H}(t) \leq x, Y_{w_{0}}=r_{2}, Y_{t}=i, Y_{t+w_{t}}=j \mid Y_{0}=r_{1}\right),
$$

we obtain the expression

$$
\frac{\left(1-\mu_{(i, j)}\left(s_{1}+s_{2} h_{i}\right)\right) \mathcal{L}\left[D_{\left(r_{1}, r_{2}\right),(i, j)}\right]\left(s_{1}, s_{2}\right)}{s_{2}\left(s_{1}+s_{2} h_{i}\right)\left(1-\mathcal{L}\left[D_{(i, j),(i, j)}\right]\left(s_{1}, s_{2}\right)\right)} p_{r_{1}, r_{2}} p_{i, j} .
$$

Case 2. $(i, j)=\left(r_{1}, r_{2}\right)$. We have

$$
\begin{aligned}
\mathrm{P}(\tilde{H}(t) & \left.\leq x, Y_{w_{0}}=r_{2}, Y_{t}=r_{1}, Y_{t+w_{t}}=r_{2} \mid Y_{0}=r_{1}\right) \\
= & \sum_{k=0}^{\infty} \mathrm{P}\left(\tilde{H}(t) \leq x, Y_{w_{0}}=r_{2}, Y_{t}=r_{1}, Y_{t+w_{t}}=r_{2}, \tau_{\left(r_{1}, r_{2}\right)}^{(k)} \leq t<\tau_{\left(r_{1}, r_{2}\right)}^{(k+1)} \mid Y_{0}=r_{1}\right) \\
= & \mathrm{P}\left(\tilde{H}(t) \leq x, Y_{t}=r_{1}, w_{0} \geq t, Y_{t+w_{t}}=r_{2} \mid Y_{0}=r_{1}\right) \\
& +\sum_{k=1}^{\infty} \mathrm{P}\left(\tilde{H}(t) \leq x, Y_{w_{0}}=r_{2}, Y_{t}=r_{1}, Y_{t+w_{t}}=r_{2}, \tau_{\left(r_{1}, r_{2}\right)}^{(k)} \leq t<\tau_{\left(r_{1}, r_{2}\right)}^{(k+1)} \mid Y_{0}=r_{1}\right) .
\end{aligned}
$$

Similarly to the arguments used in deriving (2.6) of Stefanov (2006), for the LT of

$$
\mathrm{P}\left(\tilde{H}(t) \leq x, Y_{t}=r_{1}, w_{0} \geq t, Y_{t+w_{t}}=r_{2} \mid Y_{0}=r_{1}\right),
$$

we obtain the expression

$$
\frac{\left(1-\mu_{\left(r_{1}, r_{2}\right)}\left(s_{1}+s_{2} h_{r_{1}}\right)\right)}{s_{2}\left(s_{1}+s_{2} h_{r_{1}}\right)} p_{r_{1}, r_{2}} .
$$

Therefore, the LT of

$$
\mathrm{P}\left(\tilde{H}(t) \leq x, Y_{w_{0}}=r_{2}, Y_{t}=r_{1}, Y_{t+w_{t}}=r_{2} \mid Y_{0}=r_{1}\right)
$$

is equal to

$$
\begin{aligned}
& \frac{\left(1-\mu_{\left(r_{1}, r_{2}\right)}\left(s_{1}+s_{2} h_{r_{1}}\right)\right)}{s_{2}\left(s_{1}+s_{2} h_{r_{1}}\right)} p_{r_{1}, r_{2}} \\
& \quad+\frac{\left(1-\mu_{\left(r_{1}, r_{2}\right)}\left(s_{1}+s_{2} h_{r_{1}}\right)\right) \mathcal{L}\left[D_{\left(r_{1}, r_{2}\right),\left(r_{1}, r_{2}\right)}\right]\left(s_{1}, s_{2}\right)}{s_{2}\left(s_{1}+s_{2} h_{r_{1}}\right)\left(1-\mathcal{L}\left[D_{\left(r_{1}, r_{2}\right),\left(r_{1}, r_{2}\right)}\right]\left(s_{1}, s_{2}\right)\right)} p_{r_{1}, r_{2}} p_{r_{1}, r_{2}} .
\end{aligned}
$$

In other words, this case leads to an expression that equals the sum of two components-one is the same as that in case 1 and the other corresponds to the situation when the process does not leave state $r_{1}$ before time $t$.

Putting together the expressions derived above, we obtain the statement of Theorem 4.

\section{A.1. Evaluation of the Laplace transforms $\mathscr{L}\left[D_{\left(i_{1}, j_{1}\right),\left(i_{2}, j_{2}\right)}\right]\left(s_{1}, s_{2}\right)$}

Suppose that we have an explicit, closed-form expression for the joint probability generating function, $g_{\tilde{\boldsymbol{N}}}(\boldsymbol{S})$ say, of $\tilde{N}_{i, j}\left(\tau_{\left(i_{1}, j_{1}\right),\left(i_{2}, j_{2}\right)}\right), i, j=1,2, \ldots, m$; here $\boldsymbol{S}$ is an $m \times m$ matrix. Then, applying Lemma 2, we obtain the joint LT of $\tilde{S}_{i, j}\left(\tau_{\left(i_{1}, j_{1}\right),\left(i_{2}, j_{2}\right)}\right)$ and $\tilde{N}_{i, j}\left(\tau_{\left(i_{1}, j_{1}\right),\left(i_{2}, j_{2}\right)}\right)$, where

$$
\tilde{S}_{i, j}\left(\tau_{\left(i_{1}, j_{1}\right),\left(i_{2}, j_{2}\right)}\right)=\sum_{k=1}^{\tilde{N}_{i, j}\left(\tau_{\left(i_{1}, j_{1}\right),\left(i_{2}, j_{2}\right)}\right)} Z_{(i, j)}^{(k)},
$$


and, for each $(i, j)$, the $Z_{(i, j)}^{(k)}$ are independent and identically distributed random variables with LT $\mu_{(i, j)}$. Therefore, since the reward function is a linear function of the $\tilde{S}_{i, j}\left(\tau_{\left(i_{1}, j_{1}\right),\left(i_{2}, j_{2}\right)}\right)$ and $\tilde{N}_{i, j}\left(\tau_{\left(i_{1}, j_{1}\right),\left(i_{2}, j_{2}\right)}\right)$ (recall here that $\left.\tilde{S}_{i}(t)=\sum_{j=1}^{m} \tilde{S}_{i, j}(t)\right)$, we obtain $\mathcal{L}\left[D_{\left(i_{1}, j_{1}\right),\left(i_{2}, j_{2}\right)}\right]\left(s_{1}, s_{2}\right)$.

We now explain how to derive the joint probability generating function, $g_{\tilde{N}}(\boldsymbol{S})$. Let $\hat{Y}_{n}$ be the embedded discrete-time Markov chain of the semi-Markov process $Y_{t}$. Recall that the number of states is $m$. Denote by $\tau_{\left(r_{1}, r_{2}\right), r_{3}}$ the waiting time in the Markov chain $\hat{Y}_{n}$ to move from state $r_{1}$ to state $r_{3}$, given that the next state visited after $r_{1}$ is state $r_{2}$. Let $N_{i, j}\left(\tau_{\left(r_{1}, r_{2}\right), r_{3}}\right)$ denote the number of one-step transitions from state $i$ to state $j$ in the interval $\left[0, \tau_{\left(r_{1}, r_{2}\right), r_{3}}\right]$. Let $g_{\left(r_{1}, r_{2}\right), r_{3}}(\boldsymbol{S})$ be the joint probability generating function of $N_{i, j}\left(\tau_{\left(r_{1}, r_{2}\right), r_{3}}\right), i, j=1,2, \ldots, m$. Then, using the second identity in (3), we obtain an explicit expression for this joint probability generating function. For given $i_{1}, j_{1}, i_{2}, j_{2}$, consider the following time-homogeneous multivariate Markov renewal process, $\left(C_{n}, \boldsymbol{T}_{n}\right)$, whose embedded discrete-time Markov chain, $C_{n}$, has $m+1$ states, labelled $0,1, \ldots, m$, and one-step transition probability matrix given by

$$
\left[\begin{array}{ccccc}
0 & p_{i_{2}, 1} & p_{i_{2}, 2} & \ldots & p_{i_{2}, m} \\
0 & p_{i_{2}, 1} & p_{i_{2}, 2} & \ldots & p_{i_{2}, m} \\
0 & p_{i_{2}, 1} & p_{i_{2}, 2} & \ldots & p_{i_{2}, m} \\
\vdots & \vdots & \vdots & \vdots & \vdots \\
0 & p_{i_{2}, 1} & p_{i_{2}, 2} & \ldots & p_{i_{2}, m}
\end{array}\right] .
$$

The additive component, $\boldsymbol{T}_{n}\left(=T_{n}(i, j), i, j,=1,2, \ldots, m\right)$, is $m^{2}$-dimensional and the distribution of $T_{n}-T_{n-1}$ is introduced below. For $k, r=0,1, \ldots, m$, let $G_{k, r}$ be the conditional distribution of $T_{n+1}-T_{n}$, given that $C_{n}=k$ and $C_{n+1}=r$. For $k=0$ and each $r, r=$ $1,2, \ldots, m$, the joint ( $m^{2}$-dimensional) probability generating function of $G_{k, r}$ is equal to $g_{\left(i_{1}, j_{1}\right), i_{2}}(\boldsymbol{S})$, and, for $k \geq 1$ and each $r, r=1,2, \ldots, m$, it is equal to $g_{\left(i_{2}, k\right), i_{2}}(\boldsymbol{S})$. Let $\tau_{0, j_{2}}$ be the waiting time to move from state 0 to state $j_{2}$ in the discrete-time Markov chain $C_{n}$. Again, by $N_{k, r}\left(\tau_{0, j_{2}}\right)$ we denote the number of one-step transitions of $C_{n}$ from state $k$ to state $r$ in the interval $\left[0, \tau_{0, j_{2}}\right]$; here $k, r=0,1, \ldots, m$. We can easily note the following interpretation of the $N_{k, r}\left(\tau_{0, j_{2}}\right)$. For $k \geq 1, \sum_{r=1}^{m} N_{k, r}\left(\tau_{0, j_{2}}\right)$ counts how many times, in the time interval $\left.\left[0, \tau_{\left(i_{1}, j_{1}\right),\left(i_{2}, j_{2}\right)}\right)\right]$, the semi-Markov process $Y_{t}$ enters state $i_{2}$ with $k$ being the next state visited. The joint probability generating function, say $g_{C}(\boldsymbol{s}, \boldsymbol{S})$, of the $N_{k, r}\left(\tau_{0, j_{2}}\right)$ (here the $(m+1)$-dimensional vector $\boldsymbol{s}$ corresponds to $N_{0, j}\left(\tau_{0, j_{2}}\right)$ and the $m \times m$ matrix $\boldsymbol{S}$ corresponds to $\left.N_{k, r}\left(\tau_{0, j_{2}}\right), k, r=1,2, \ldots, m\right)$ is derived using the second identity in (3).

Finally, in view of Lemma 5, below, we obtain the following identity for the joint probability generating function $g_{\tilde{N}}(S)$ :

$$
g_{\tilde{\boldsymbol{N}}}(\boldsymbol{S})=g_{C}(\tilde{\boldsymbol{S}}(\boldsymbol{S}), \tilde{\boldsymbol{S}}(\boldsymbol{S})),
$$

where the $(m+1)$-dimensional vector $\tilde{\boldsymbol{S}}(\boldsymbol{S})$ and the $m \times m$-matrix $\tilde{\boldsymbol{S}}(\boldsymbol{S})$ are given by

$$
\begin{gathered}
\tilde{\boldsymbol{S}}(\boldsymbol{S})=\left(1, g_{\left(i_{1}, j_{1}\right), i_{2}}(\boldsymbol{S}), g_{\left(i_{1}, j_{1}\right), i_{2}}(\boldsymbol{S}), \ldots, g_{\left(i_{1}, j_{1}\right), i_{2}}(\boldsymbol{S})\right), \\
\tilde{\boldsymbol{S}}(\boldsymbol{S})(k, r)=g_{\left(i_{2}, k\right), i_{2}}(\boldsymbol{S}), \quad k, r=1,2, \ldots, m,
\end{gathered}
$$

and $g_{\left(r_{1}, r_{2}\right), r_{3}}(\boldsymbol{S})$ is introduced above.

Lemma 5. Let $\boldsymbol{\Gamma}=\left(\gamma_{1}, \gamma_{2}, \ldots, \gamma_{r}\right)^{\top}$ be a vector of nonnegative integer-valued random variables with joint probability generating function $g_{\Gamma}\left(s_{1}, s_{2}, \ldots, s_{r}\right)$. Let $\boldsymbol{Z}_{n}^{(i)}, i=1,2, \ldots, r$, be $r$ independent sequences of independent and identically distributed random vectors (with the same dimension, $u$ say) that are independent of $\boldsymbol{\Gamma}$. For each $n$, it is assumed that the distribution 
of $\boldsymbol{Z}_{n}^{(i)}$ follows the distribution of a random vector, $\boldsymbol{Z}^{(i)}$ say, for $i=1,2, \ldots, r$. Also, it is assumed that, for each $i$, the components of $\boldsymbol{Z}^{(i)}$ are nonnegative integers and the joint probability generating function of $\boldsymbol{Z}^{(i)}$ is denoted by $g_{Z^{(i)}}\left(\boldsymbol{t}^{(i)}\right)$, where $\boldsymbol{t}^{(i)}=\left(t_{1}^{(i)}, t_{2}^{(i)}, \ldots, t_{u}^{(i)}\right)^{\top}$ is a u-dimensional vector. Let

$$
\boldsymbol{U}_{i}=\sum_{j=1}^{\gamma_{i}} \boldsymbol{Z}_{j}^{(i)}, \quad i=1,2, \ldots, r .
$$

Then the joint probability generating function of the ru-dimensional vector

$$
\boldsymbol{U}=\left(\boldsymbol{U}_{1}, \boldsymbol{U}_{2}, \ldots, \boldsymbol{U}_{r}\right)^{\top}
$$

is equal to

$$
g_{\boldsymbol{U}}\left(\boldsymbol{t}^{(1)}, \boldsymbol{t}^{(2)}, \ldots, \boldsymbol{t}^{(r)}\right)=g_{\boldsymbol{\Gamma}}\left(g_{\boldsymbol{Z}^{(1)}}\left(\boldsymbol{t}^{(1)}\right), g_{\boldsymbol{Z}^{(2)}}\left(\boldsymbol{t}^{(2)}\right), \ldots, g_{\boldsymbol{Z}^{(r)}}\left(\boldsymbol{t}^{(r)}\right)\right) .
$$

Proof. The proof is an easy extension of Lemma 2.1 of Stefanov (2000b) and Lemma 2 to the case of multidimensional summands.

\section{References}

AвAte, J. AND Whitt, W. (2006). A unified framework for numerically inverting Laplace transforms. INFORMS J. Computing 18, 408-421.

Abate, J., Choudhury, G. L. And Whitt, W. (1998). Numerical inversion of multidimensional Laplace transforms by the Laguerre method. Performance Evaluation 31, 229-243.

Asmussen, S. AND PIHLSGÅRD, M. (2004). Transient properties of many-server queues and related QBDs. Queueing Systems 4, 249-270.

Ball, F. and Stefanov, V. T. (2005). Evaluation of identity-by-descent probabilities for half-sibs on continuous genome. Math. Biosci. 196, 215-225.

Ball, F., Milne, R. K. and Yeo, G. F. (1994). Continuous-time Markov chains in a random environment, with applications to ion channel modelling. Adv. Appl. Prob. 26, 919-946.

Bickeboller, H. And Thompson, E. A. (1996a). Distribution of genome shared IBD by half-sibs: approximation by the Poisson clumping heuristic. Theoret. Pop. Biol. 50, 66-90.

Bickeboller, H. AND Thompson, E. A. (1996b). The probability distribution of the amount of an individual's genome surviving to the following generation. Genetics 143, 1043-1049.

Browning, S. (2000). A Monte Carlo approach to calculating probabilities for continuous identity by descent data. J. Appl. Prob. 37, 850-864.

Cannings, C. (2003). The identity by descent process along the chromosome. Human Heredity 56, 126-130.

Choudhury, G. L., Lucantoni, D. M., And Whitt, W. (1994). Multidimensional transform inversion with applications to the transient $M / G / 1$ queue. Ann. Appl. Prob. 4, 719-740.

Donnelly, K. P. (1983). The probability that related individuals share some section of genome identical by descent. Theoret. Pop. Biol. 23, 34-63.

Gaver, D. P., Jacobs, P. A. ANd Latouche, G. (1984). Finite birth-and-death models in randomly changing environments. Adv. Appl. Prob. 16, 715-731.

Iosifescu, M. (1980). Finite Markov Processes and Their Applications. John Wiley, Chichester.

Karlin, S. And Taylor, H. M. (1981). A Second Course in Stochastic Processes. Academic Press, New York.

LANGe, K. (2002). Mathematical and Statistical Methods for Genetic Analysis, 2nd edn. Springer, New York.

Latouche, G. ANd Ramaswami, V. (1999). Introduction to Matrix Analytic Methods in Stochastic Modeling. Society for Industrial and Applied Mathematics, Philadelphia, PA.

LI, Q.-L. AND CAO, J. (2004). Two types of $R G$-factorizations of quasi-birth-and-death processes and their applications to stochastic integral functionals. Stoch. Models 20, 299-340.

Lucantoni, D. M., Choudhury, G. L. And Whitt, W. (1994). The transient BMAP/G/1 Queue. Commun. Statist. Stoch. Models 10, 145-182.

Stefanov, V. T. (2000a). Distribution of genome shared IBD by two individuals in grandparent-type relationship. Genetics 156, 1403-1410.

Stefanov, V. T. (2000b). On some waiting time problems. J. Appl. Prob. 37, 756-764. 
Stefanov, V. T. (2002). Statistics on continuous IBD data: exact distribution evaluation for a pair of full(half)-sibs and a pair of a (great-)grandchild with a (great-)grandparent. BMC Genetics 3, No. 7.

Stefanov, V. T. (2004). Distribution of the amount of genetic material from a chromosomal segment surviving to the following generation. J. Appl. Prob. 41, 345-354. (Correction: 41 (2004), 1248-1249)

Stefanov, V. T. (2006). Exact distributions for reward functions on semi-Markov and Markov additive processes. J. Appl. Prob. 43, 1053-1065.

Thompson, E. A. (2000). Statistical Inference From Genetic Data on Pedigrees (NSF-CBMS Regional Conf. Ser. Prob. Statist. 6). American Statistical Association, Alexandria, VA.

Walters, K. And Cannings, C. (2005). The probability density of the total IBD length over a single autosome in unilineal relationships. Theoret. Pop. Biol. 68, 55-63. 